\title{
Diet Quality Indices Used in Australian and New Zealand Adults: A Systematic Review and Critical Appraisal
}

\author{
Hlaing Hlaing-Hlaing ${ }^{1,2, *}$, Kristine Pezdirc ${ }^{1,2}$, Meredith Tavener ${ }^{1,2}$, Erica L. James ${ }^{1,2}(\mathbb{D}$ and \\ Alexis Hure ${ }^{1,2}$ D \\ 1 School of Medicine and Public Health, University of Newcastle, Callaghan, Newcastle, NSW 2308, Australia; \\ kristine.pezdirc@newcastle.edu.au (K.P.); meredith.tavener@newcastle.edu.au (M.T.); \\ erica.james@newcastle.edu.au (E.L.J.); alexis.hure@newcastle.edu.au (A.H.) \\ 2 Hunter Medical Research Institute, New Lambton Heights, Newcastle, NSW 2305, Australia \\ * Correspondence: HlaingHlaingHlaing@uon.edu.au
}

Received: 19 November 2020; Accepted: 7 December 2020; Published: 9 December 2020

check for updates

\begin{abstract}
Distilling the complexity of overall diet into a simple measure or summative score by data reduction methods has become a common practice in nutritional epidemiology. Recent reviews on diet quality indices (DQI) have highlighted the importance of sound construction criteria and validation. The aim of this current review was to identify and critically appraise all DQI used within Australian and New Zealand adult populations. Twenty-five existing DQI were identified by electronic searching in Medline and hand searching of reference lists. DQI were constructed based on the respective national dietary guidelines and condition-specific recommendations. For preferable features of DQI, six captured the dimensions of adequacy, moderation and balance; five had a nested structure; 12 consisted of foods, food groups and nutrients; 11 used metric scoring systems and most of those with metric scales used normative cutoff points. Food frequency questionnaires, either alone or with other methods, were the most common dietary assessment method used in 20 DQI. For evaluation of DQI, construct validity and relative validity are reported. Based on our critical appraisal, Dietary Guideline Index (DGI), Dietary Guideline Index-2013 (DGI-2013), Total Diet Score (TDS), Healthy Eating Index for Australian Adults-2013 (HEIFA-2013), and Aussie-Diet Quality Index (Aussie-DQI) were the preferred DQI used in Australian adults according to dimension, indicator selection, scoring criteria and evaluation. Further work is needed to enhance the construction of all Australian and New Zealand DQI, especially in terms of dimension and structure, for alignment with recommended construction criteria.
\end{abstract}

Keywords: adults; dietary assessment; diet quality; evaluation; index; systematic review; validity

\section{Introduction}

In public health and epidemiology, there is often a need to condense the complex nature of dietary patterns and intake into a simple measure or summative score [1-3]. This is typically done in one of two ways. Data reduction techniques, like principal component, factor or cluster analysis, can be used to derive dietary patterns from comprehensive dietary data through multivariate analysis $[4,5]$. Diet quality indices (DQI) are the alternative, which apply a priori scoring criteria for adherence to dietary recommendations, like dietary guidelines $[4,6,7]$. Diet quality (DQ) can then be used to quantify chronic disease risk and mortality across population [3]. In the present review we focus on predefined DQI that aims to summarize the overall diet into a single measure.

To evaluate people's adherence to accepted guidelines and optimal eating behavior, DQI are developed [3]. The earliest DQI were constructed with reference to the American Dietary 
Guidelines [8-10] and the Mediterranean diet [11]. Other indices have been derived in accordance with country-specific dietary guidelines or modified based on previously developed indices.

Several reviews on DQI have been published [1-3,6,7,12]. The review by Waijer et al. [1] emphasized methodological issues of DQI for their compositions, scoring and validity. Another review [3] highlighted the importance of validation studies by means of biomarkers or intermediate-risk factors for potential applicability in both clinical and public health settings. Two recent reviews have focused on relevant index construction criteria $[13,14]$. Burggraf et al. discussed the contribution of a theoretical basis, including all dimensions of diet quality (adequacy, moderation, variety and balance) and current diet-health relationship knowledge in DQI construction [14]. A further recent systematic review conducted by Trijsburg et al. identified those DQI developed for both low- and middle-income countries (LMIC) and high-income countries [13]. The authors concluded that there is a need for sound metrics to assess diet quality and suggested the guidelines for DQI construction both in LMIC and high-income countries [13].

Both Burggraf et al. and Trijsburg et al. have reviewed some, but not all, of the DQI [8,11,15-23] that have been used in Australian and New Zealand adults, especially with consideration given to the construction methodology [14], age group and context [13]. However, these Australian and New Zealand tools are in among many other international tools, derived from slightly different international dietary guidelines on which they are based. Therefore, this review aims to systematically identify and critically appraise all DQI used specifically with Australian and New Zealand adults. The construction criteria developed by Burggraf et al. and supplemented by Trijsburg et al. [13,14] was used to critically appraise each DQI and identify those that performed best. Diet-disease associations were considered only for validity assessment.

\section{Materials and Methods}

The review process was conducted by applying methods recommended by the Center for Reviews and Dissemination (CRD), University of York [24] and the Preferred Reporting Items for Systematic Reviews and Meta-Analyses (PRISMA) statement [25]. The review protocol was registered with the PROSPERO, registration number CRD42020149720.

\subsection{Developing the Search Strategy and Databases to Be Included}

The electronic database search was conducted in Medline, Embase and Cumulative Index to Nursing and Allied Health Literature (CINAHL) for papers published between 2000 to 2019 . The keywords used in the review were "diet*, healthy eating, food, nutri" ${ }^{*}$ in combination with AND for "index, score, tool, indic*", then combined with "AND" for "Australia or New Zealand" either in the title, abstract, subject headings or original title. Filters were used to limit the results to those conducted in the human and English language. In addition, the reference lists of the retrieved papers were hand-searched to identify the relevant studies that were not detected by the electronic search strategy.

\subsection{Inclusion/Exclusion Criteria for Eligible Studies}

Studies were included if (1) participants were human adults aged 18 years and over, (2) participants were based in Australia and/or New Zealand, (3) DQ was assessed by using indices based on latest or current nutritional guidelines or recommendations, (4) scores, indices or tools that summarize the overall diet into a single value, (5) study designs were observational or experimental, (6) they described the development, application and/or validity of the DQ measure by means of nutrient adequacy, biomarkers or health outcomes.

Studies were excluded if (1) conducted in animals, (2) conducted among children, adolescents, pregnant and/or lactating women, (3) used a posteriori or empirically derived dietary pattern by using factor analysis (principal component analysis) or cluster analysis, (4) scores, indices or tools focused on one or more nutrients (e.g., fat), but not the overall diet, (5) the study design was a review, commentary, editorial, conference proceeding or theses, and (6) non-English publications. 


\subsection{Screening Procedure}

Titles of all articles in Medline, Embase and CINAHL were first screened by a single reviewer (HHH). After hand searching the references from Medline, Embase and CINAHL, only one additional paper was found. Therefore, duplicate screening was restricted to the Medline database only (See in Figure 1). Eligibility was assessed by two independent reviewers (HHH, KP) based on information contained in the title, abstract and description/MESH headings. If eligibility was unclear, the paper was referred to a third reviewer $(\mathrm{AH})$ and discussed to reach a consensus.

Based on the information contained in the title and abstract, relevance to the review was assessed first. Articles were excluded by using a hierarchical approach based on population, the topic of interest, context and study design. The full texts were retrieved and screened to check for compliance with eligibility. Some were excluded according to the inclusion and exclusion criteria. The reference lists of the articles were also examined for possible inclusion in the review. Then the second round of screening was conducted by the first two reviewers, and the eligibility of the publication was considered. Only the studies deemed eligible were data extracted.

\subsection{Quality of the Evidence}

The quality of each article was assessed by two independent reviewers (HHH and KP) using the American Dietetic Association Quality Criteria Checklist for primary research [26]. The Checklist addressed issues of relevance to practice and validity for scientific soundness. For each point on the Checklist, each reviewer assigned 'Yes' if the criterion was met, ' $\mathrm{No}^{\prime}$ if the criterion was not met, 'Unclear' if the criterion was not clearly described, or 'N/A' if the criterion did not apply to the study. The answers were tabulated, and each study was rated as "positive", "negative," or "neutral". Those articles assigned "yes" to six or more of the validity questions were considered methodologically rigorous and rated as "positive". If the article was assigned "no" or "unclear" on at least one of the validity questions, it was rated as "neutral". Articles assigned "no" or "unclear" on six or more of the validity questions were considered not meeting methodological quality and rated as "negative" (See in Table S1). Any discrepancy between the reviewers was resolved through discussion.

\subsection{Data Extraction}

Two independent reviewers (HHH, KP) extracted relevant data using a data extraction form designed specifically for this review, adapted from the Cochrane Review Group [27]. Critical appraisal of the included DQI was conducted by using construction criteria developed by Burggraf et al. and supplemented by Trijsburg et al. [13,14]. The features of DQI assessed in our study were based on theoretical framework; dimensions (adequacy, moderation, balance, variety); dimensional structure (unordered, ordered, nested); indicator selection (database and component types- food groups, nutrients or both); normalization methods which contain scaling procedure (dichotomous, ordinal, metric) and cutoff values (normative, percentile, uniform, group-specific); and aggregation technique (unweighted, weighted) [14], the inclusion of healthy or unhealthy components, and evaluation of DQI [13]. Preferable features of DQI were the inclusion of adequacy, moderation and balance in dimension; nested structure; both food groups and nutrients in indicator selection; both healthy and unhealthy components; metric scaling procedure; normative cutoff points; weighted aggregation technique; and evaluation of DQI. Disagreement between reviewers $(\mathrm{HHH}, \mathrm{KP})$ was resolved by discussion and/or assessment by a third reviewer $(\mathrm{AH})$.

The data extracted include information about the overview of the most current DQI (ordered chronologically) (Table 1); overview of components of DQI (Table S2); summary findings of studies investigating DQI, health outcomes and non-health-related factors (Table 2) and critical appraisal of DQI by previously suggested recommendations (Table 3). 

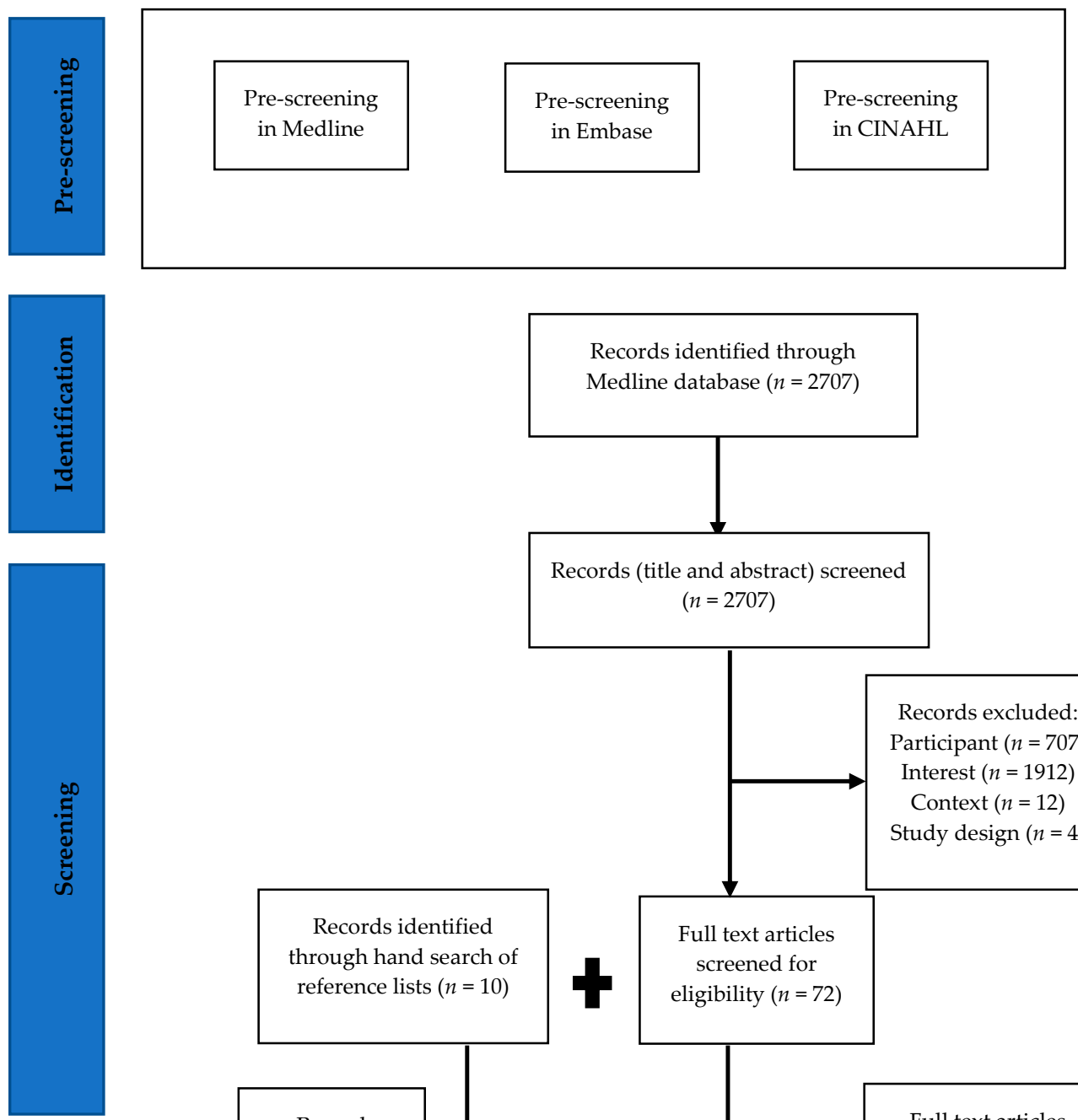
$(n=2707)$

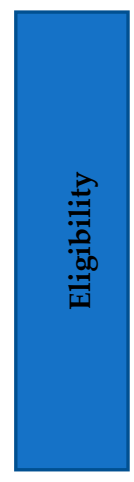

rough hand search of reference lists $(n=10)$
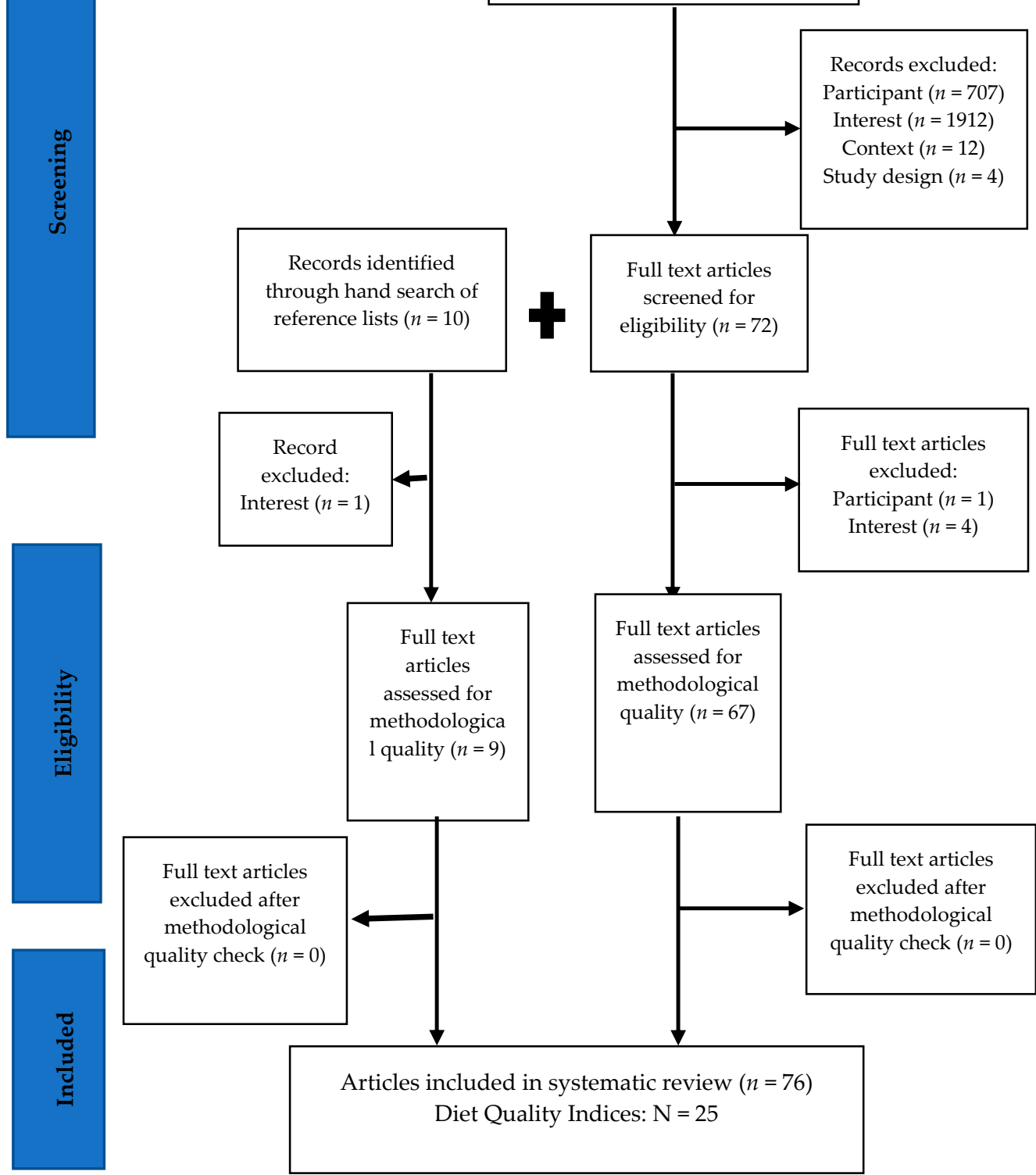

Articles included in systematic review $(n=76)$ Diet Quality Indices: N = 25

Figure 1. Flow chart for the systematic review process. 


\section{Results}

\subsection{Australian and New Zealand Diet Quality Indices}

In total, 76 relevant articles describing 25 DQI were identified. Of the 25 indices identified, 24 had been applied in Australia [8,11,15-19,21-23,28-41] and one in New Zealand [20]. Thirty-six percent ( $n=9 / 25)$ were modified versions of an original tool, leaving 16 different indices, as summarized in Table 1.

Nearly half of the DQI $(n=12)$ aimed to operationalize the Australian Dietary Guidelines [16-19,28-30,33,36-38,42] and one operationalized New Zealand's Food and Nutrition Guidelines for Healthy Adults [43]. A further three indices [8,31,41] were based on US dietary guidelines [44-46]. Nine of the identified indices operationalized condition-specific recommendations for a Mediterranean diet [11,21,39,47], chronic diseases [48-52], lowering blood pressure [53], cardiovascular disease prevention [54] and anti-inflammation [34]. Among recent Australian DQI $(n=8)$, $50 \%$ of their original indices $[19,28,29,33]$ had been modelled or adapted from US indices $[9,10,41,55-58]$.

The included DQI has been used across a variety of adults, based on sex and age. Of all of the DQI, five were validated and/or applied with women only $[15,29,31,37,41]$ and 20 of them with both men and women $[8,11,16-23,28,30,32,33,35,36,38-40,47]$. Furthermore, the DQI was validated and/or applied across a broad age range of adults aged 18 and above $(n=12)$, or more specifically, middle-aged adults $(n=5)$, older adults $(n=4)$, middle-aged and older adults $(n=2)$, young university adults $(n=1)$ or a mean age was reported $(n=2)$. 
Table 1. Overview of the most current diet quality indices used in Australian and New Zealand adults.

\begin{tabular}{|c|c|c|c|c|c|c|}
\hline Index & Reference & $\begin{array}{c}\text { Theoretical or } \\
\text { Epidemiological Basis }\end{array}$ & $\begin{array}{l}\text { Original Tool (Local } \\
\text { or International) }\end{array}$ & $\begin{array}{l}\text { Modified or Adapted } \\
\text { Intermediate Tool }\end{array}$ & Components & $\begin{array}{l}\text { Evaluation of Diet } \\
\text { Quality Index }\end{array}$ \\
\hline \multicolumn{7}{|c|}{ Based on Australian Dietary Guidelines } \\
\hline $\begin{array}{l}\text { Australian Healthy } \\
\text { Eating Index } \\
\text { (Aust-HEI) }\end{array}$ & $\begin{array}{c}\text { Australian Institute of } \\
\text { Health and Welfare, } \\
2007 \text { [28] }\end{array}$ & $\begin{array}{c}\text { Australian Guide to } \\
\text { Healthy Eating } \\
\text { (AGHE), 1998 [59], } \\
\text { Dietary Guidelines } \\
\text { for Australian Adults, } \\
2003 \text { [60] }\end{array}$ & - & $\begin{array}{l}\text { Variety score from } \\
\text { previous food variety } \\
\text { score [56] and } \\
\text { Diet Quality } \\
\text { Index-Revised [57]; } \\
\text { Healthy choice score } \\
\text { from Recommended } \\
\text { Food Score [41] }\end{array}$ & $\begin{array}{l}\text { Variety; } \\
\text { healthy choices; fruit; } \\
\text { vegetable; low-fat } \\
\text { milk; trim fat meat; } \\
\text { high saturated fat, } \\
\text { low nutrient } \\
\text { density food }\end{array}$ & $\begin{array}{c}\text { Nutrient intakes, } \\
\text { demographic } \\
\text { and lifestyle } \\
\text { characteristics, } \\
\text { general health status }\end{array}$ \\
\hline $\begin{array}{c}\text { Australian } \\
\text { Recommended Food } \\
\text { Score-1 (ARFS-1) }\end{array}$ & Collins et al. 2015 [42] & $\begin{array}{l}\text { Australian Dietary } \\
\text { Guidelines-2013 [61] }\end{array}$ & $\begin{array}{l}\text { Recommended Food } \\
\text { Score [55] }\end{array}$ & $\begin{array}{c}\text { Australian } \\
\text { Recommended Food } \\
\text { Score [29], Australian } \\
\text { Child and Adolescent } \\
\text { Recommended Food } \\
\text { Score [62] }\end{array}$ & $\begin{array}{l}\text { Vegetable; fruit; } \\
\text { protein foods; grains; } \\
\text { dairy; fats; alcohol }\end{array}$ & Nutrient intakes \\
\hline $\begin{array}{l}\text { Commonwealth } \\
\text { Scientific and } \\
\text { Industrial Research } \\
\text { Organization- } \\
\text { Healthy Diet Score } \\
\text { (CSIRO HDS) }\end{array}$ & Hendrie et al. 2017 [63] & $\begin{array}{l}\text { Australian Dietary } \\
\text { Guidelines-2013 [61] }\end{array}$ & - & $\begin{array}{l}\text { Dietary Guideline } \\
\text { Index [19] }\end{array}$ & $\begin{array}{l}\text { Variety; vegetables; } \\
\text { fruits; whole-grain } \\
\text { cereals; meat and } \\
\text { alternatives; dairy } \\
\text { and alternatives; } \\
\text { water; discretionary } \\
\text { foods; trim fat; fats } \\
\text { and oils; salt; sugar; } \\
\text { alcohol }\end{array}$ & $\begin{array}{c}\text { Mean dietary } \\
\text { score component }\end{array}$ \\
\hline Total Diet Score (TDS) & Russell et al. 2013 [33] & $\begin{array}{c}\text { Australian Guide to } \\
\text { Healthy Eating } \\
\text { (AGHE), 1998 [59], } \\
\text { Dietary Guidelines } \\
\text { for Australian Adults, } \\
2003 \text { [60] }\end{array}$ & $\begin{array}{l}\text { US } 2005 \text { Dietary } \\
\text { Guidelines } \\
\text { Adherence Index [58] }\end{array}$ & $\begin{array}{l}\text { Australian Healthy } \\
\text { Eating Index [28] }\end{array}$ & $\begin{array}{l}\text { Vegetables, legumes } \\
\text { and fruit; } \\
\text { cereals/whole grains; } \\
\text { lean meats and } \\
\text { alternatives; } \\
\text { dairy and alternatives; } \\
\text { saturated fat; sodium; } \\
\text { alcohol; sugar; extra } \\
\text { food; physical activity }\end{array}$ & All-cause mortality \\
\hline
\end{tabular}


Table 1. Cont.

\begin{tabular}{|c|c|c|c|c|c|c|}
\hline Index & Reference & $\begin{array}{c}\text { Theoretical or } \\
\text { Epidemiological Basis }\end{array}$ & $\begin{array}{l}\text { Original Tool (Local } \\
\text { or International) }\end{array}$ & $\begin{array}{l}\text { Modified or Adapted } \\
\text { Intermediate Tool }\end{array}$ & Components & $\begin{array}{c}\text { Evaluation of Diet } \\
\text { Quality Index }\end{array}$ \\
\hline $\begin{array}{l}\text { Aussie-Diet Quality } \\
\text { Index (Aussie-DQI) }\end{array}$ & Zarrin et al. 2013 [18] & $\begin{array}{c}\text { Australian Guide to } \\
\text { Healthy Eating } \\
\text { (AGHE), 1998 [59], } \\
\text { Dietary Guidelines } \\
\text { for Australian Adults, } \\
\text { 2003 [60] }\end{array}$ & $\begin{array}{c}\text { Australia National } \\
\text { Health Priority Area } \\
\text { (ANHPA) [64] }\end{array}$ & $\begin{array}{l}\text { Australian Healthy } \\
\text { Eating Index [28], } \\
\text { Dietary Guideline } \\
\text { Index [19] }\end{array}$ & $\begin{array}{l}\text { Vegetables; fruits; } \\
\text { dairy products; } \\
\text { meat and alternatives; } \\
\text { cereals; saturated fat; } \\
\text { sugar; alcohol; } \\
\text { processed meat; } \\
\text { salt/sodium; variety }\end{array}$ & $\begin{array}{l}\text { Sociodemographic } \\
\text { and lifestyle } \\
\text { characteristics, } \\
\text { cancer mortality }\end{array}$ \\
\hline $\begin{array}{l}\text { Healthy Eating Index } \\
\text { for Australian-2013 } \\
\text { (HEIFA-2013) }\end{array}$ & Roy et al. 2016 [16] & $\begin{array}{l}\text { Australian Dietary } \\
\text { Guidelines-2013 [61] }\end{array}$ & - & - & $\begin{array}{l}\text { Discretionary foods; } \\
\text { vegetables; fruits; } \\
\text { whole grains; } \\
\text { protein foods; dairy; } \\
\text { water; saturated fat; } \\
\text { sodium; sugar; } \\
\quad \text { alcohol }\end{array}$ & Nutrient intakes \\
\hline $\begin{array}{c}\text { Australian Diet } \\
\text { Quality Score (ADQS) }\end{array}$ & Froud et al. 2019 [30] & $\begin{array}{l}\text { Recommended Daily } \\
\text { Intake (RDI) of } \\
\text { the Australian } \\
\text { Dietary Guidelines } \\
\text { (not specified) }\end{array}$ & - & - & $\begin{array}{l}\text { Vegetable; fruits; } \\
\text { whole grains; } \\
\text { processed grains; } \\
\text { dairy; proteins; nuts; } \\
\text { seafood; fats ratio; } \\
\text { extras ratio }\end{array}$ & Nil \\
\hline \multicolumn{7}{|c|}{ Based on New Zealand Dietary Guidelines } \\
\hline $\begin{array}{l}\text { Healthy Dietary } \\
\text { Habits Index (HDHI) }\end{array}$ & Wong et al. 2017 [20] & $\begin{array}{l}\text { New Zealand food } \\
\text { and nutrition } \\
\text { guidelines for healthy } \\
\text { adults [43] }\end{array}$ & $\begin{array}{l}\text { Healthy Dietary } \\
\text { Habit Score for } \\
\text { New Zealand } \\
\text { adolescents [65] }\end{array}$ & - & $\begin{array}{l}\text { Red meat; chicken; } \\
\text { fish/shellfish; milk; } \\
\text { spread; low-fat foods; } \\
\text { fries; bread; fruits; } \\
\text { vegetable; soft drinks; } \\
\text { breakfast; fast foods; } \\
\text { added salt; } \\
\text { low salt food }\end{array}$ & $\begin{array}{l}\text { Nutrient intakes, } \\
\text { biomarker }\end{array}$ \\
\hline
\end{tabular}


Table 1. Cont.

\begin{tabular}{|c|c|c|c|c|c|c|}
\hline Index & Reference & $\begin{array}{c}\text { Theoretical or } \\
\text { Epidemiological Basis }\end{array}$ & $\begin{array}{c}\text { Original Tool (Local } \\
\text { or International) }\end{array}$ & $\begin{array}{l}\text { Modified or Adapted } \\
\text { Intermediate Tool }\end{array}$ & Components & $\begin{array}{c}\text { Evaluation of Diet } \\
\text { Quality Index }\end{array}$ \\
\hline \multicolumn{7}{|c|}{ Based on US Dietary Guidelines } \\
\hline $\begin{array}{c}\text { Diet Quality } \\
\text { Index-Revised } \\
\text { (DQI-R) }\end{array}$ & Haines et al. 1999 [8] & $\begin{array}{c}\text { 1989-Dietary } \\
\text { recommendations } \\
\text { from the US National } \\
\text { Academy of Sciences } \\
\text { and Dietary } \\
\text { Guidelines for } \\
\text { Americans [44], } \\
\text { dietary reference } \\
\text { intakes [45] }\end{array}$ & $\begin{array}{l}\text { Diet Quality } \\
\text { Index [10] }\end{array}$ & - & $\begin{array}{l}\text { Total fat; saturated fat; } \\
\text { cholesterol; fruits; } \\
\text { vegetables; grains; } \\
\text { calcium; iron; } \\
\text { diversity; moderation }\end{array}$ & Nutrient intakes \\
\hline $\begin{array}{l}\text { Recommended Food } \\
\text { Score (RFS) }\end{array}$ & $\begin{array}{c}\text { Kant and Graubaud, } \\
2000[41]\end{array}$ & $\begin{array}{l}\text { 1989-Dietary } \\
\text { recommendations } \\
\text { from the US National } \\
\text { Academy of Sciences } \\
\text { and Dietary } \\
\text { Guidelines for } \\
\text { Americans [44], } \\
\text { epidemiological } \\
\text { evidence [46] }\end{array}$ & $\begin{array}{l}\text { Developed by Kant } \\
\text { and Graubaud [41] }\end{array}$ & - & $\begin{array}{l}\text { Fruits; vegetables; } \\
\text { whole grains; } \\
\text { lean meat } \\
\text { or alternatives; } \\
\text { low-fat dairy }\end{array}$ & Mortality \\
\hline $\begin{array}{l}\text { Not Recommended } \\
\text { Food Score (NRFS) }\end{array}$ & Michels et al. 2002 [31] & $\begin{array}{c}\text { 1989-Dietary } \\
\text { recommendations } \\
\text { from the US National } \\
\text { Academy of Sciences } \\
\text { and Dietary } \\
\text { Guidelines for } \\
\text { Americans [44], } \\
\text { epidemiological } \\
\text { evidence [46] }\end{array}$ & $\begin{array}{l}\text { US Dietary guidelines } \\
\text { and results of large } \\
\text { epidemiological } \\
\text { studies [31] }\end{array}$ & - & $\begin{array}{l}\text { Meat and its products; } \\
\text { fried food; foods high } \\
\text { in fat; others }\end{array}$ & Mortality \\
\hline
\end{tabular}


Table 1. Cont.

\begin{tabular}{|c|c|c|c|c|c|c|}
\hline Index & Reference & $\begin{array}{c}\text { Theoretical or } \\
\text { Epidemiological Basis }\end{array}$ & $\begin{array}{c}\text { Original Tool (Local } \\
\text { or International) }\end{array}$ & $\begin{array}{l}\text { Modified or Adapted } \\
\text { Intermediate Tool }\end{array}$ & Components & $\begin{array}{c}\text { Evaluation of Diet } \\
\text { Quality Index }\end{array}$ \\
\hline \multicolumn{7}{|c|}{ Specific Dietary Pattern Recommendations } \\
\hline $\begin{array}{l}\text { Mediterranean Diet } \\
\text { Scale (MDS) }\end{array}$ & $\begin{array}{c}\text { Trichopoulou et al. } \\
2005 \text { [39] }\end{array}$ & & $\begin{array}{c}\text { Assessment of } \\
\text { adherence to a } \\
\text { Mediterranean diet } \\
\text { developed by } \\
\text { Trichopoulou et al. [21] }\end{array}$ & - & $\begin{array}{c}\text { Grains; vegetables; } \\
\text { nuts and legumes; } \\
\text { fruits; fish; olive oil; } \\
\text { dairy products; red } \\
\text { and processed meat; } \\
\text { alcohol }\end{array}$ & Mortality \\
\hline $\begin{array}{l}\text { Alternative Healthy } \\
\text { Eating Index-2010 } \\
\text { (AHEI-2010) }\end{array}$ & Chiuve et al. 2012 [22] & $\begin{array}{l}\text { Foods and nutrients } \\
\text { that lowered chronic } \\
\text { diseases based } \\
\text { on the Mediterranean } \\
\text { diet [48-52] }\end{array}$ & $\begin{array}{l}\text { Healthy Eating } \\
\text { Index [9] }\end{array}$ & $\begin{array}{l}\text { Alternative Healthy } \\
\text { Eating Index } \\
\text { (AHEI) [23] }\end{array}$ & $\begin{array}{l}\text { Vegetables; fruits; } \\
\text { nuts and soy protein; } \\
\text { ratio of white to red } \\
\text { meat; cereal fiber; } \\
\text { trans-fat; ratio of } \\
\text { polyunsaturated to } \\
\text { saturated fatty acids; } \\
\text { alcohol; } \\
\text { multivitamin use }\end{array}$ & Chronic disease risk \\
\hline $\begin{array}{l}\text { Diet Quality Tool } \\
\text { (DQT) }\end{array}$ & $\begin{array}{c}\text { O'Reilly et al. } 2012 \\
\text { [32] }\end{array}$ & $\begin{array}{c}\text { Heart Foundation's } \\
\text { secondary prevention } \\
\text { nutrition guidelines } \\
{[54]}\end{array}$ & - & - & $\begin{array}{l}\text { Vegetable; fruits; rice, } \\
\text { pasta or noodle; } \\
\text { breakfast cereals; } \\
\text { bread; spread; milk; } \\
\text { trim fat meat; } \\
\text { takeaway meals; } \\
\text { discretionary foods; } \\
\text { fish; salt use }\end{array}$ & Nutrient intakes \\
\hline $\begin{array}{l}\text { Dietary Inflammatory } \\
\text { Index (DII) }\end{array}$ & Shivappa et al. 2014 [66] & $\begin{array}{c}\text { Literature-derived, } \\
\text { population-based } \\
\text { dietary inflammatory } \\
\text { index [34] }\end{array}$ & Original DII [67] & - & $\begin{array}{l}\text { Nutrients, spices, } \\
\text { whole food and other }\end{array}$ & $\begin{array}{l}\text { High-sensitivity } \\
\text { C-reactive protein }\end{array}$ \\
\hline
\end{tabular}




\subsection{Composition of Diet Quality Indices}

The composition of the Australian and New Zealand DQI in this review included food groups, individual foods and beverages, nutrients, variety, and/or occasionally other lifestyle behaviors, such as physical activity and supplement use (Table S2). Thirteen indices were comprised exclusively of food and/or food groups $[17,19,20,28,29,31,32,36,37,41,42,47,63]$ and twelve indices consisted of foods, food groups and nutrients $[8,11,15,16,18,21-23,30,34,39,68]$.

Almost all indices $(n=23)$ included vegetable intake, ranging from 22 vegetable items represented by ARFS and 7 captured by RFS. Twenty-two indices included fruits, with 14 fruit items represented by ARFS and 7 by RFS. Whole grains or whole grain cereals were included in 14 indices [15-17,19,20,22,29,30,32,33,36,38,41,42], but units of measurement were different. For source of protein foods, meat and/or its alternatives were included in 23 out of 25 DQI (92\%); of which lean meats was counted in five DQI $[16,17,19,33,41]$, and gave points as proportion $[17,19]$ or servings per day [16] or per week [33]. Twelve of the DQI included fish in their scoring [11,20,21,29,32,33,36,38,39,41,42,47], and nearly half of them $(n=5)$ gave points $[11,21,33,39,47]$. Majority of the indices $(n=20,80 \%)$, except NRFS, DQI-R, AHEI, AHEI-2010 and DII, comprised of dairy products, in which $30 \%$ of them $(6 / 20)$ recorded low-fat dairy $[15,16,41]$ or low-fat/skimmed milk $[28,29,33]$ and another $30 \%(6 / 20)$ recorded type of milk $[17,19,20,32,36,63]$. The remaining DQI $(n=8,32 \%)$ did not specify dairy or milk as low-fat.

Fourteen of the DQI included points for alcohol [11,16-19,21-23,29,33,34,36,38,39], however there was not agreement on the level of intake that was recommended. Almost all of the DQI $(n=13)$, except ARFS, considered alcohol to be part of a positive diet quality, and nearly half the tools counted alcohol intake in terms of range [11,18,21-23,33]. Fluid intake, including water, tea and coffee, was measured in DQI $(n=10)$, such as HEIFA-2013 [16] and DGI-2013 [17]. Furthermore, all Australian Dietary Guideline-based indices $(n=12)$ were composed of discretionary foods; $50 \%$ of them $(6 / 12)$ included jam, ice-cream or chocolate $[16,19,29,36,38,42]$, while $33 \%$ $(4 / 12)$ included salt [17-19,36], sugar [16-18,33], confectionary [16,19,36,38] and sugar-sweetened beverages $[16,19,36,38]$. The remaining $17 \%(2 / 12)$ included take away food [38] or meat products [18,38] or fried food [36,38] or non-specified extra food $[19,68]$ or extra ratios [30].

The most commonly found macronutrient in DQI was fat. Twenty percent $(n=5)$ of the DQI $[8,16,18,33,34]$ were composed of saturated fatty acid (SFA); $12 \%(n=3)$ were composed of trans-fatty acids [22,23,34] or PUFA [16,22,34]; $8 \%(n=2)$ were composed of MUFA [16,34], total fat [8,34] or cholesterol [8,34]. In addition, fat ratio was included in 4 DQI; such as the ratio of MUFA to SFA [21,47], ratio of PUFA to SFA [23] and proportion of unsaturated fats to SFA [30]. Protein was included in two DQI such as DII [34] and ADQS [30]. Moreover, micronutrients such as sodium $(n=4)[15,16,18,22]$, other minerals $(n=2)[8,34]$ and vitamin $(n=1)$ [34] were included as components of DQI.

In addition to food and nutrients, $36 \%(9 / 25)$ of the DQI included dietary variety or diversity $[8,16-19,28,33,37,38]$; and one DQ index $(4 \%)$ included dietary moderation as a separate component [8]. Lifestyle behaviors, such as physical activity [33] and multivitamin use [23], were also included. Cooking practices such as salt use $(n=6)$ and trimming of fat either before or after cooking $(n=7)$ were considered in the development of DQ indices. Likewise, eating patterns such as fast food or takeaway consumption $(n=3)[20,32,38]$, breakfast consumption $(n=1)$ [20] and ratio of energy intake to energy expenditure $(n=1)$ [33] were included.

\subsection{Scoring of the Diet Quality Indices}

The items within each DQ index were typically classified as recommended to be included as part of a healthy diet, or to be limited. Some indices assessed DQ by including foods that are recommended as well as those that should be limited $(n=21)$, while others focused on recommended foods only $(n=3)$ [29,41,42], or foods to limit only $(n=1)$ [31] (refer to Table S3). The items included in the DQI were quantified with different measurement units such as servings [8,15-20,22,23,28,32,33,36-38], grams [11,18,21-23,30,33,39,47], milligrams [15,16,18,22,33], 
standard drinks [22,36], proportions [16,17,19,30,36-38], \% energy [8,16,18,22,23,33], \% recommended dietary allowance (\% RDA) [8], ratio [21,23,33,47], type of food item $[17,19,20,28,32,36,38]$, frequency $[16,17,19,20,28,32,36,38]$, reported consumption [29,31,41,42], mean (sd) value from global database [34] and kilo Joules [30].

For scoring, 13 out of $25 \mathrm{DQI}(52 \%)$ used specific cutoffs for minimum and maximum intakes for each component and then calculated the intermediate proportional score $[8,16-19,22,23,28,32,33,36-38]$; five DQI $(25 \%)$ used median or quintile intakes of the sample [11,15,21,39,47]; four DQI used the reported consumption of any amount of the component, or alternatively no consumption [29,31,41,42]; one DQ index based on the effect of the food parameter on inflammation [34], one DQ index based on DHQ responses [20], one DQ index by combining recommended dietary intake (RDI) and mean intake [30].

\subsection{Dietary Assessment Methods Used}

Generally, in nutritional epidemiology studies, dietary intake was measured by using real-time recording methods such as (weighted) food record and duplication method, and recall methods such as diet history, food frequency questionnaire (FFQ) and 24-h recall (24-h R) [69]. Food frequency questionnaire alone was the predominant method of dietary assessment in derivations of DQI $(n=13)$. Together with FFQ other dietary assessment methods were used in validation studies of DQI; 24-h Rs [18,19,39]; and weighed food records (WFRs) [16,31]. Short dietary assessment tools were also used in DQI development and/or evaluation; 38-item short food survey (SFS) [38], 24-item short dietary question (12-item FFQ and 12-item dietary behavior questions) [36] and 13-item question [32]. Furthermore, a 25-item dietary habits questionnaire and multiple-pass 24-h R were used in HDHI [20].

\subsection{Evaluation of Diet Quality Indices}

The DQI were evaluated in various ways. Reproducibility, the index's ability to yielding similar outcomes on two different occasions [42] and reliability and internal consistency $[28,63]$ were assessed. Content validity, which is the ability of DQ index items to reflect all contents or aspects it is supposed to measure [20], and construct validity by exploring the relationships between DQI and sociodemographic, health and behavioral characteristics, food and nutrient intakes $[17-20,28,29,32,66]$ was also evaluated. Additionally, relative or criterion validity was evaluated by investigating the agreement of DQ scores between two different dietary assessment methods and predicting mortality or morbidity $[8,16,18,20,32,42,63,68]$.

\subsection{Summary Findings of Studies Investigating Diet Quality Indices, Health Outcomes and Non-Health Related Factors}

Table 2 summarizes the major finding of the publications that used the DQI. The most frequently used DQI are ARFS $(n=12)$ [29,70-80], DGI-2013 $(n=11)$ [17,81-90], TDS $(n=10)$ [33,68,91-99], DGI $(n=9) \quad[19,70,91,100-105]$, DII $(n=9) \quad$ [106-114] and Mediterranean diet-based indices $(n=8)[71,72,88,89,106-108,115]$. Some studies $(n=9)$ used two or more DQI in observing association between DQ and health related outcomes [70-72,84,88,89,106-108]. Health related outcomes or measurements or biomarkers observed in the articles using reviewed DQI were anthropometric measurements $(n=8)$; depression $(n=5)$; diabetes mellitus or abnormal glucose metabolism $(n=4)$; cardiometabolic risk factor or hypertension $(n=5)$, mortality $(n=3)$, cancer $(n=3)$, overweight or obesity $(n=3)$, quality of life or functional status or psychological function $(n=3)$, telomere length or aging $(n=2)$, sensory impairment $(n=2)$, vascular dysfunction $(n=2)$, chronic kidney disease $(n=1)$, asthma $(n=1)$ and inflammatory marker $(n=1)$.

The studies reported that there were relationships between high DQ scores and favorable health related outcomes. Negative associations were found between high $\mathrm{DQ}$ and all-cause mortality $\left(\mathrm{HR}_{\mathrm{Q} 5}\right.$ vs. Q1:0.79; 95\% CI: 0.63, 0.98; $\left.\mathrm{P}_{\text {trend }}=0.04\right)$ [33], total mortality $\left(\mathrm{HR}_{\mathrm{Q} 5}\right.$ vs. $\mathrm{Q} 1: 0.86 ; 95 \%$ CI: $0.80-0.93$; $\left.\mathrm{P}_{\text {trend }}<0.0001\right)$ [108] and cancer mortality $\left(\mathrm{HR}_{\mathrm{T} 3}\right.$ vs. $\left.{ }_{\mathrm{T} 1}: 0.3 ; 95 \% \mathrm{CI}: 0.11,0.83 ; \mathrm{P}_{\text {trend }}=0.06\right)$ [18]. 
However, there was inconsistent findings for associations between DQ and risk of overweight or obesity [74-76]; diabetes mellitus or abnormal glucose metabolism [70,97,100,116]; cancer [106,107,110]; and depression $[30,72,73]$.

Non-health related factors associated with DQ were sociodemographic characteristics such as sex $[19,36,117]$, age $[19,20,35,77,101,117]$, education $[17,77,103]$, occupation $[17,86,101]$, income $[19,103]$, socioeconomic status $[19,20,29,37,83,103,117]$ and residence [17]. Moreover, lifestyle factors such as smoking [17,19,20,36,117], alcohol consumption [20], physical activity [17,36,77,101], nutrition knowledge [80,118], cooking meals for oneself [91], number of meals shared [35], meal frequency [81] and takeaway meal consumption [91,119] were found to be associated with DQ.

\subsection{Critical Appraisal of Diet Quality Indices by Previous Suggested Recommendations}

The DQI $[8,11,15-23,28-34,36-39,41,42,47]$ were described and shaded gray according to their adherence to the recommended points developed by Burggraf et al. and Trijsburg et al. (Table 3). Sixteen of the 25 DQI were constructed to reflect the respective dietary guidelines, and the remaining indices measured condition-specific recommendations. Almost all the DQI $(n=21)$, except ARFS, ARFS-1, RFS and NRFS, captured the dimensions of adequacy (foods that people should eat more of) and moderation (foods that people should limit). Dietary variety was considered in almost half of the indices $(n=12)$. Nearly one-fourth of the indices included the dimensions of adequacy, moderation and balance $[11,21-23,30,47]$. With respect to the dimensional structure, only five indices had a nested structure $[16,17,19,33,36]$, but ten indices had an ordered structure $[8,18,21,29,37,39,41,42,47,63]$. As database or dietary assessment method for construction of DQI, food frequency questionnaires, either alone or with other dietary assessment methods, were mostly used $(n=20)$. Twelve indices consisted of foods, food groups and nutrients $[8,11,15,16,18,21-23,30,33,34,47]$. The DQI used different scoring systems; metric $(n=11)$, dichotomous $(n=8)$ and ordinal $(n=6)$. Most of the DQI with metric scales used normative cutoff for minimum and maximum intakes for each component and then calculated the intermediate proportional score, for example, DGI, Aussie-DQI, AEHI-2010. Based on evaluation of DQI, construct validity $[17-20,28,29,32,66]$, relative validity $[8,16,18,20,32,42,63,68]$, content validity [20], reproducibility [42], reliability and internal consistency $[28,63]$ were reported. 
Table 2. Summary of findings from studies investigating diet quality indices, health outcomes and non-health-related factors.

\begin{tabular}{|c|c|c|c|c|c|c|}
\hline Index & Reference & $\begin{array}{l}\text { Validation Status of } \\
\text { Diet Quality Index }\end{array}$ & Population Used in & $\begin{array}{l}\text { Dietary Assessment } \\
\text { Methods Used in } \\
\text { Publications }\end{array}$ & $\begin{array}{l}\text { Health-Related } \\
\text { Outcomes }\end{array}$ & Summary of Findings \\
\hline $\begin{array}{l}\text { Australian Healthy } \\
\text { Eating Index } \\
\text { (Aust-HEI) }\end{array}$ & $\begin{array}{l}\text { Forsyth, } 2012 \text { [120] } \\
\text { Forsyth, } 2015 \text { [121] }\end{array}$ & $\begin{array}{c}\text { Tested construct } \\
\text { validity [28] }\end{array}$ & $\begin{array}{c}\text { Adults aged } \geq 18 \\
\text { years with depression } \\
\text { and anxiety }[120,121]\end{array}$ & $\begin{array}{c}\text { Diet History } \\
\text { Questionnaire }[120,121]\end{array}$ & $\begin{array}{l}\text { Depression, Anxiety } \\
\text { and Stress Scale } \\
\text { (DASS) }[120,121]\end{array}$ & $\begin{array}{l}\text { Mean total Aust-HEI was } 42.8 \\
\text { (range 20-60), and Aust-HEI and } \\
\text { DASS were negatively correlated } \\
(p<0.001) \text { [120]. Improved DASS } \\
\text { in the diet and physical activity } \\
\text { intervention group }(p<0.05)[121] .\end{array}$ \\
\hline $\begin{array}{c}\text { Australian } \\
\text { Recommended Food } \\
\text { Score (ARFS) }\end{array}$ & $\begin{array}{c}\text { Collins, } 2008 \text { [29] } \\
\text { Collins, } 2011 \text { [79] } \\
\text { Morrison, } 2012 \text { [77] } \\
\text { Aljadani, } 2013 \text { [74] } \\
\text { Aljadani, } 2013 \text { [75] } \\
\text { Alhazmi, } 2014 \text { [70] } \\
\text { Potter, } 2014 \text { [78] } \\
\text { Petersen, } 2015 \text { [71] } \\
\text { Aljadani, } 2016 \text { [76] } \\
\text { Kullen, 2016 [80] } \\
\text { Lai, 2016 [72] } \\
\text { Lai, 2017 [73] }\end{array}$ & $\begin{array}{l}\text { Tested construct } \\
\text { validity [29] }\end{array}$ & $\begin{array}{c}\text { Adults aged } \geq 50 \\
\text { years [71]; mid-aged } \\
\text { women ( } 50-55 \text { years) } \\
{[29,70,72-74,76,78,79] ;} \\
\text { young women (mean } \\
\text { age: } 27.6 \pm 1.5 \text { years } \\
\text { and } 34.2 \pm 5.1 \text { years) } \\
{[75,77] ; \text { young men }} \\
\text { (mean age: } \\
28.7 \pm 8.9 \text { years) [80] }\end{array}$ & $\begin{array}{l}\text { FFQ (74-item food } \\
\text { and 6-item } \\
\text { alcohol) }[29,70-80]\end{array}$ & $\begin{array}{c}\text { Diabetes [70]; } \\
\text { Depression [72,73]; } \\
\text { Overweight or } \\
\text { obese [74-76]; } \\
\text { Diet quality } \\
\text { [29,71,77-80] }\end{array}$ & $\begin{array}{c}\text { No association between ARFS and } \\
\text { diabetes risk [70]. } \\
\text { Women who maintained moderate } \\
\text { or high ARFS scores had a low } \\
\text { risk of } \\
\text { depression ( } p=0.045 \text { and } 0.01 \text { ) [73], } \\
\text { but no longitudinal association } \\
\text { between ARFS and depressive } \\
\text { symptoms [72]. } \\
\text { Association between ARFS and } \\
\text { overweight or obesity is not } \\
\text { consistent [74-76]. } \\
\text { Factors associated with higher } \\
\text { ARFS were socioeconomic status, } \\
\text { education, marital status, smoking, } \\
\text { physical activity (all } p<0.0001) \\
\text { [29]; age, education, physical } \\
\text { activity (all } p<0.001) \text { [77]; } \\
\text { nutrition knowledge } \\
(p=0.009) \text { [80]. }\end{array}$ \\
\hline $\begin{array}{c}\text { Australian } \\
\text { Recommended Food } \\
\text { Score-1 (ARFS-1) }\end{array}$ & $\begin{array}{c}\text { Baker, } 2014 \text { [122] } \\
\text { O' Brien, } 2014 \text { [123] } \\
\text { Collins, } 2015[42] \\
\text { Ashton, } 2017 \text { [124] } \\
\text { Ashton, } 2017 \text { [125] } \\
\text { Williams, } 2017 \text { [35] } \\
\text { Ashton, } 2018 \text { [126] } \\
\text { Harbury, } 2019 \text { [118] }\end{array}$ & $\begin{array}{l}\text { Tested reproducibility, } \\
\text { comparative } \\
\text { validity [42]; relative } \\
\text { validity [124] }\end{array}$ & $\begin{array}{c}\text { Adults aged } \geq 16 \\
\text { years [35], } \geq 18 \text { years } \\
\text { [118,122-124,126], } \\
\geq 30 \text { years [42]; } \\
\text { young men aged } \\
18-25 \text { years [125] }\end{array}$ & $\begin{array}{l}\text { Subset of } 70 \text { items } \\
\text { from } 120 \text {-item FFQ } \\
\text { [42,118,122-126]; } \\
\text { Healthy Eating Quiz } \\
\text { (online survey, } \\
70 \text { items) [35] }\end{array}$ & $\begin{array}{l}\text { Plasma carotenoid [124]; } \\
\text { Weight loss [123]; } \\
\text { Diet quality } \\
{[42,118,122,125,126] .}\end{array}$ & $\begin{array}{l}\text { Significant correlation between } \\
\text { total ARFS- } 1 \text { and plasma } \\
\text { tota carotenoids } \\
(\mathrm{r}=0.17, p<0.05)[124] . \\
\text { The intervention groups } \\
\text { significantly lost more weight than } \\
\text { the control group after } 12 \text {-weeks } \\
\quad(p<0.001) \text { [123]. } \\
\text { Factors associated with ARFS-1 } \\
\text { were nutrition knowledge and BMI } \\
\quad(p<0.001) \text { [118]. }\end{array}$ \\
\hline
\end{tabular}


Table 2. Cont

\begin{tabular}{|c|c|c|c|c|c|c|}
\hline Index & Reference & $\begin{array}{l}\text { Validation Status of } \\
\text { Diet Quality Index }\end{array}$ & Population Used in & $\begin{array}{l}\text { Dietary Assessment } \\
\text { Methods Used in } \\
\text { Publications }\end{array}$ & $\begin{array}{l}\text { Health-Related } \\
\text { Outcomes }\end{array}$ & Summary of Findings \\
\hline $\begin{array}{l}\text { Dietary Guideline } \\
\text { Index (DGI) }\end{array}$ & $\begin{array}{c}\text { McNaughton, } \\
\text { 2008 [19] } \\
\text { McNaughton, } \\
\text { 2009 [100] } \\
\text { Arabshahi, 2011 [101] } \\
\text { Arabshahi, } 2012 \text { [102] } \\
\text { Thorpe, } 2013 \text { [91] } \\
\text { Alhazmi, } 2014 \text { [70] } \\
\text { Backholer, } 2016 \text { [103] } \\
\text { Olstad, 2017 [104] } \\
\text { Smith, } 2017 \text { [105] }\end{array}$ & $\begin{array}{l}\text { Tested construct } \\
\text { validity [19] }\end{array}$ & $\begin{array}{c}\text { Adults aged } \\
\geq 19 \text { years [19], } \\
\text { 18-36 years [91], } \\
\geq 25 \text { years [100-103], } \\
\text { 26-36 years [105]; } \\
\text { mid-aged women } \\
\text { (50-55 years) [70], } \\
\text { women } \\
18-46 \text { years [104] }\end{array}$ & $\begin{array}{c}\text { FFQs: } 74 \text {-item [100], } \\
\text { FFQ (74-item food } \\
\text { and 6-item } \\
\text { alcohol) [70,103], } \\
\text { 107-item [91], } \\
\text { 151-item [101,102], } \\
\text { items not } \\
\text { mentioned [104]; } \\
\text { FFQ and others: } \\
\text { 127-item FFQ and } \\
\text { food habit } \\
\text { questionnaire } \\
\text { (FHQ) [105]; 108-item } \\
\text { FFQ, single 24-h R [19] }\end{array}$ & $\begin{array}{c}\text { Diabetes [70,100] and } \\
\text { cardiometabolic risk } \\
\text { factors [100]; } \\
\text { Anthropometric } \\
\text { measurements [102,104]; } \\
\text { Diet quality } \\
\text { [19,91,101,103,105] }\end{array}$ & 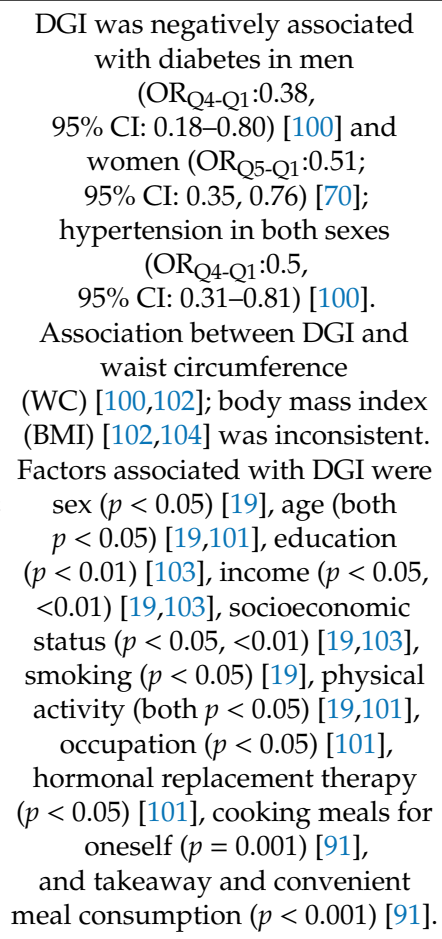 \\
\hline $\begin{array}{l}\text { Modified Dietary } \\
\text { Guideline Index } \\
\text { (Modified DGI) }\end{array}$ & McLeod, 2011 [37] & Not tested & $\begin{array}{c}\text { Women }(\text { mean } \\
\text { age }=32.3 \text { years) }[37]\end{array}$ & 137-item FFQ [37] & Diet quality [37] & $\begin{array}{l}\text { Diet quality was significantly } \\
\text { better in women of a high } \\
\text { socioeconomic group as compared } \\
\text { to those of the low socioeconomic } \\
\text { group }(p<0.001)[37] .\end{array}$ \\
\hline
\end{tabular}


Table 2. Cont

\begin{tabular}{|c|c|c|c|c|c|c|}
\hline Index & Reference & $\begin{array}{l}\text { Validation Status of } \\
\text { Diet Quality Index }\end{array}$ & Population Used in & $\begin{array}{l}\text { Dietary Assessment } \\
\text { Methods Used in } \\
\text { Publications }\end{array}$ & $\begin{array}{l}\text { Health-Related } \\
\text { Outcomes }\end{array}$ & Summary of Findings \\
\hline $\begin{array}{l}\text { Dietary Guideline } \\
\text { Index-2013 } \\
\text { (DGI-2013) }\end{array}$ & $\begin{array}{c}\text { Milte et al. } 2015 \text { [89] } \\
\text { Livingstone, } 2016 \text { [84] } \\
\text { Thorpe, } 2016 \text { [17] } \\
\text { Leech, } 2016 \text { [81] } \\
\text { Leech, } 2017 \text { [82] } \\
\text { Livingstone, } 2017 \text { [83] } \\
\text { Martin, } 2017 \text { [86] } \\
\text { Ribeiro, } 2017 \text { [90] } \\
\text { Livingstone, } 2018 \text { [85] } \\
\text { Milte, } 2018 \text { [88] } \\
\text { Martin, } 2019 \text { [87] }\end{array}$ & $\begin{array}{l}\text { Tested construct } \\
\text { validity [17] }\end{array}$ & $\begin{array}{c}\text { Adults aged } \\
\geq 19 \text { years [81-85], } \\
\text { 55-68 years [17,88,89]; } \\
\text { women aged } \\
\text { 18-50 years [86,87]; } \\
\text { men aged } \\
\geq 74 \text { years [90] }\end{array}$ & $\begin{array}{c}\text { FFQ }(74 \text {-item food and } \\
\text { 6-item alcohol) [86,87], } \\
\text { 111-item FFQ and } \\
\text { food-related behavior } \\
\text { questions }[17,88,89] ; \\
\text { two 24-h Rs [81-85]; } \\
\text { diet histories } \\
\text { questionnaire [90] }\end{array}$ & $\begin{array}{c}\text { Obesity }[82,84,85,90] \\
\text { Hypertension [84]; } \\
\text { health related quality } \\
\text { of life (QOL) [89]; } \\
\text { Telomere length [88]; } \\
\text { Diet quality } \\
\text { [17,81,83,86,87] }\end{array}$ & $\begin{array}{c}\text { Higher DGI-2013 scores were } \\
\text { negatively associated with obesity } \\
\text { measured by BMI (both } \\
\left.\mathrm{P}_{\text {trend }}<0.05\right)[84,85], \text { WC (both } \\
\left.\mathrm{P}_{\text {trend }}<0.05\right)[84,85] \text {, waist-hip } \\
\text { ratio (WHR) }(p<0.001) \text { [90]. } \\
\text { Men with higher DGI-2013 were } \\
\text { less likely to be hypertensive } \\
\left.\text { ( } \mathrm{P}_{\text {trend }}<0.05\right) \text { [84]. } \\
\text { Higher DGI-2013 scores were } \\
\text { associated with better } \\
\text { health-related QOL }(p<0.05) \text { [89]. } \\
\text { No association between DGI-2013 } \\
\text { and relative telomere length [88]. } \\
\text { Factors associated with DGI-2013 } \\
\text { were sex }(p<0.001), \text { residence } \\
\text { (men, } p<0.001) \text { [17], occupation } \\
(\text { men: } p=0.02 ; \text { women: } p=0.043) \\
\text { [17,86], income (women: } p=0.013) \\
\text { [86], education }(p<0.001) \text { [17], } \\
\text { socioeconomic status } \\
\left(\mathrm{P}_{\text {trend }}<0.001\right) \text { [83], smoking } \\
(p<0.001)[17], \text { physical activity } \\
(p<0.001) \text { [17], } \\
\text { BMI ( } p<0.001) \text { [17], frequency of } \\
\text { meals }(p<0.001) \text { [81]. }\end{array}$ \\
\hline
\end{tabular}


Table 2. Cont

\begin{tabular}{|c|c|c|c|c|c|c|}
\hline Index & Reference & $\begin{array}{l}\text { Validation Status of } \\
\text { Diet Quality Index }\end{array}$ & Population Used in & $\begin{array}{l}\text { Dietary Assessment } \\
\text { Methods Used in } \\
\text { Publications }\end{array}$ & $\begin{array}{l}\text { Health-Related } \\
\text { Outcomes }\end{array}$ & Summary of Findings \\
\hline $\begin{array}{c}\text { RESIDential } \\
\text { Environments } \\
\text { (RESIDE) Dietary } \\
\text { Guideline } \\
\text { Index (RDGI) }\end{array}$ & Bivoltsis, 2018 [36] & Not tested & $\begin{array}{c}\text { Adults aged } \\
\geq 25 \text { years }[36]\end{array}$ & $\begin{array}{l}\text { 24-item questionnaire } \\
\text { (12 from validated } \\
\text { FFQ, } 12 \text { from } \\
\text { validated dietary } \\
\text { behavior } \\
\text { questions) [36] }\end{array}$ & Diet quality [36] & $\begin{array}{c}\text { Two simple RESIDE dietary } \\
\text { guideline indices using subsets of } \\
\text { six survey items (S-RDGI1), } \\
\text { and nine survey items (S-RDGI2) } \\
\text { showed reasonable agreement } \\
\text { with RDGI (Spearman rho }=0.78, \\
\text { 0.84). For all indices, higher diet } \\
\text { quality was associated with sex (all } \\
p<0.001), \text { age (S-RDGI1 and } \\
\text { S-RDGI2, } p<0.001 \text { ), smoking } \\
\text { status (S-RDGI1: } p=0.001 \text {, SRDGI } \\
\text { and S-RDGI2: } p<0.001), \text { physical } \\
\text { activity (RDGI: } p=0.001, \text { S-RDGI1 } \\
p<0.0001, \text { S-RDGI2: } \\
p=0.002 \text { ) [36]. }\end{array}$ \\
\hline $\begin{array}{c}\text { Commonwealth } \\
\text { Scientific and } \\
\text { Industrial Research } \\
\text { Organization Healthy } \\
\text { Diet Score } \\
\text { (CSIRO HDS) }\end{array}$ & $\begin{array}{c}\text { Hendrie, } 2017 \text { [63] } \\
\text { Hendrie, } 2017 \text { [38] } \\
\text { Hendrie, } 2018 \text { [127] }\end{array}$ & $\begin{array}{l}\text { Tested reliability and } \\
\text { relative validity [63] }\end{array}$ & $\begin{array}{c}\text { Adults aged } \\
\geq 18 \text { years }[38,127], \\
\text { aged } 19-50 \text { years }[63]\end{array}$ & $\begin{array}{c}\text { 38-item SFS [38,127]; } \\
\text { 38-item SFS and three } \\
\text { 24-h Rs [63] }\end{array}$ & $\begin{array}{c}\text { Obesity [127]; } \\
\text { Diet quality }[38,63]\end{array}$ & $\begin{array}{c}\text { Adults having a lower score were } \\
\text { more likely to obese }\left(\mathrm{OR} \mathrm{Q}_{\mathrm{Q} 1-\mathrm{Q} 5} 2.99\right. \\
\text { CI: } 2.88,3.11)[127] . \\
\text { Women scored higher than men } \\
(59.9 \pm 12.6 \text { vs. } 56.2 \pm 13.1) \\
\text { older adults higher than younger } \\
\text { adults (>71yr: } 63.1 \pm 11.7 \text { vs. } 18-30 \\
\text { yr: } 57.3 \pm 13.2), \text { and normal-weight } \\
\text { adults higher than obese adults } \\
\text { (60.5 } \pm 12.6 \text { vs. } 55.7 \pm 13.2)[38] .\end{array}$ \\
\hline
\end{tabular}


Table 2. Cont

\begin{tabular}{|c|c|c|c|c|c|c|}
\hline Index & Reference & $\begin{array}{l}\text { Validation Status of } \\
\text { Diet Quality Index }\end{array}$ & Population Used in & $\begin{array}{l}\text { Dietary Assessment } \\
\text { Methods Used in } \\
\text { Publications }\end{array}$ & $\begin{array}{l}\text { Health-Related } \\
\text { Outcomes }\end{array}$ & Summary of Findings \\
\hline Total Diet Score (TDS) & $\begin{array}{c}\text { Russell, } 2013 \text { [33] } \\
\text { Gopinath, } 2013 \text { [96] } \\
\text { Gopinath, } 2013 \text { [95] } \\
\text { Gopinath, } 2013 \text { [97] } \\
\text { Gopinath, } 2014 \text { [98] } \\
\text { Gopinath, } 2014 \text { [92] } \\
\text { Hong, 2014 [94] } \\
\text { Gopinath, } 2016 \text { [93] } \\
\text { Roach, } 2017 \text { [99] } \\
\text { Russell, } 2017 \text { [68] }\end{array}$ & $\begin{array}{l}\text { Tested criterion } \\
\text { validity [33] }\end{array}$ & $\begin{array}{c}\text { Adults aged } \\
\geq 49 \text { years [33,93,94], } \\
\geq 50 \text { years [95-98], } \\
\geq 55 \text { years [92], } \\
65-85 \text { years [68], } \\
\text { median } \\
\text { age-72 years [99] }\end{array}$ & $\begin{array}{c}\text { 145-item } \\
\text { FFQ [33,92-98], } \\
\text { 145-item FFQ and } \\
\text { 4-day WFRs [68], } \\
\text { three 24 h Rs and } \\
\text { PUFA FFQ [99] }\end{array}$ & $\begin{array}{c}\text { All-cause } \\
\text { mortality [33]; } \\
\text { Chronic kidney } \\
\text { disease (CKD) [96], } \\
\text { visual impairment [94], } \\
\text { retinal vascular } \\
\text { change [95], quality of } \\
\text { life (QOL) [92], } \\
\text { aging [93] } \\
\text { Impaired fasting } \\
\text { glucose (IFG) and } \\
\text { diabetes [97], } \\
\text { dual sensory } \\
\text { impairment (DSI) [98], } \\
\text { Diet quality [68,99] }\end{array}$ & 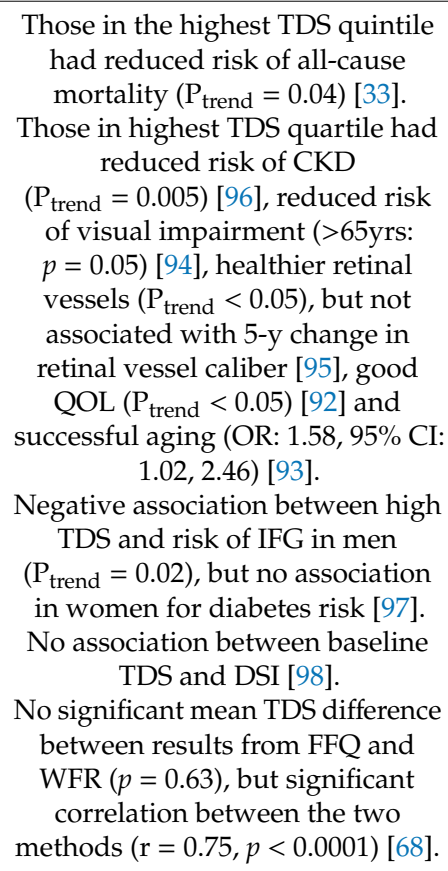 \\
\hline $\begin{array}{l}\text { Aussie-Diet Quality } \\
\text { Index (Aussie-DQI) }\end{array}$ & Zarrin, 2013 [18] & $\begin{array}{l}\text { Tested content, } \\
\text { construct and } \\
\text { criterion validity [18] }\end{array}$ & $\begin{array}{c}\text { Adults aged } \geq 19 \\
\text { years from } 1995 \\
\text { National Nutrition } \\
\text { Survey (NNS); aged } \\
\geq 25 \text { from the } \\
\text { Nambour Skin Cancer } \\
\text { Study (NSC) [18] }\end{array}$ & $\begin{array}{c}\text { 129-item FFQ and a } \\
\text { 24-h R [18] }\end{array}$ & $\begin{array}{c}\text { All-cause and cancer } \\
\text { mortality [18] }\end{array}$ & $\begin{array}{c}\text { Higher Aussie-DQI scores were } \\
\text { associated with higher desirable } \\
\text { nutrient intakes and inversely } \\
\text { associated with risk of cancer } \\
\text { mortality in men (HR: } 0.3,95 \% \mathrm{CI} \text { : } \\
0.11,0.83)[18] .\end{array}$ \\
\hline
\end{tabular}


Table 2. Cont.

\begin{tabular}{|c|c|c|c|c|c|c|}
\hline Index & Reference & $\begin{array}{l}\text { Validation Status of } \\
\text { Diet Quality Index }\end{array}$ & Population Used in & $\begin{array}{l}\text { Dietary Assessment } \\
\text { Methods Used in } \\
\text { Publications }\end{array}$ & $\begin{array}{l}\text { Health-Related } \\
\text { Outcomes }\end{array}$ & Summary of Findings \\
\hline $\begin{array}{l}\text { Healthy Eating Index } \\
\text { for Australian } \\
\text { Adults-2013 } \\
\text { (HEIFA-2013) }\end{array}$ & $\begin{array}{c}\text { Roy, } 2016[16] \\
\text { Roy, } 2017 \text { [119] } \\
\text { Grech, } 2017[117] \\
\text { Grech, } 2017 \text { [128] }\end{array}$ & $\begin{array}{l}\text { Tested criterion } \\
\text { validity and internal } \\
\text { consistency [16] }\end{array}$ & $\begin{array}{l}\text { Adults aged } 18-34 \\
\text { years [16,117,128], } \\
19-24 \text { years [119] }\end{array}$ & $\begin{array}{c}\text { FFQ (74-item food } \\
\text { and 6-item alcohol) } \\
\text { and 5-d WFR [16], } \\
\text { validated mobile } \\
\text { application (e-DIA } \\
\text { app) [119], two 24-h } \\
\text { Rs [117,128] }\end{array}$ & $\begin{array}{c}\text { Diet quality } \\
\text { [16,117,119]; } \\
\text { Dietary energy } \\
\text { density [128] }\end{array}$ & $\begin{array}{l}\text { Positive correlation of essential } \\
\text { micronutrients between both FFQ } \\
\text { and WFR HEIFA-2013 scores } \\
\quad\left(\mathrm{P}_{\text {trend }}<0.0005,\right. \\
\text { Cronbach } \alpha=0.41)[16] . \\
\text { Higher HEIFA-2013 was associated } \\
\text { with reduced university campus } \\
\text { and other takeaway foods } \\
\text { consumption }\left(\mathrm{P}_{\text {trend }}<0.001\right), \mathrm{BMI} \\
\left(\mathrm{P}_{\text {trend }}=0.02\right) \text { and } \mathrm{WC} \\
\left(\mathrm{P}_{\text {trend }}=0.05\right)[119] ; \\
\text { sociodemographic and lifestyle } \\
\text { characteristics }(p<0.05)[117] . \\
\text { Higher dietary energy density was } \\
\text { associated with lower HEIFA-2013 } \\
(p<0.0001)[128] .\end{array}$ \\
\hline $\begin{array}{c}\text { Australian Diet } \\
\text { Quality Score (ADQS) }\end{array}$ & Froud, 2019 [30] & Not tested & $\begin{array}{c}\text { Adults aged } 18-75 \\
\text { years [30] }\end{array}$ & $\begin{array}{l}\text { FFQ (74-item food } \\
\text { and 6-item } \\
\text { alcohol) [30] }\end{array}$ & Depression [30] & $\begin{array}{l}\text { Lower ADQS was associated with } \\
\text { increased depression risk } \\
(p=0.037)[30] .\end{array}$ \\
\hline $\begin{array}{l}\text { Healthy Dietary } \\
\text { Habits Index (HDHI) }\end{array}$ & $\begin{array}{c}\text { Wong, } 2017 \text { [20] } \\
\text { Davison, } 2017 \text { [129] }\end{array}$ & $\begin{array}{l}\text { Tested content, } \\
\text { construct and } \\
\text { criterion validity [20] }\end{array}$ & $\begin{array}{c}\text { Adults aged } \\
\geq 19 \text { years [20], } \\
\text { child-parent pairs } \\
\text { (mean age of } \\
\text { child }=10.2 \text { years, } \\
\text { parent }=41.6 \text { years) }[129]\end{array}$ & $\begin{array}{l}\text { Two 24-h Rs and } \\
\text { 25-item DHQ [20], } \\
\text { Children; 28-item } \\
\text { FFQ and Parents; } \\
\text { 25-item DHQ [129] }\end{array}$ & Diet quality $[20,129]$ & $\begin{array}{l}\text { Higher HDHI score was associated } \\
\text { with sociodemographic and } \\
\text { lifestyle characteristics; higher } \\
\text { nutrient intakes (all } p<0.001) \text { [20] } \\
\text { Parental DQI score was associated } \\
\text { with a child's dietary pattern score } \\
\qquad(p<0.001) \text { [129]. }\end{array}$ \\
\hline $\begin{array}{l}\text { Diet Quality } \\
\text { Index-Revised } \\
\text { (DQI-R) }\end{array}$ & Reeves et al. 2013 [116] & $\begin{array}{l}\text { Tested reproducibility } \\
\text { and validity [57] }\end{array}$ & $\begin{array}{c}\text { Adults aged } \\
\geq 25 \text { years [116] }\end{array}$ & 74-item FFQ [116] & $\begin{array}{l}\text { AGM- Abnormal } \\
\text { glucose metabolism } \\
\text { (IFG, impaired } \\
\text { glucose tolerance, } \\
\text { diabetes) [116] }\end{array}$ & $\begin{array}{l}\text { Women with low DQI-R were } \\
\text { more likely to have AGM } \\
\left(\mathrm{P}_{\text {trend }}=0.012\right)[116] .\end{array}$ \\
\hline
\end{tabular}


Table 2. Cont.

\begin{tabular}{|c|c|c|c|c|c|c|}
\hline Index & Reference & $\begin{array}{l}\text { Validation Status of } \\
\text { Diet Quality Index }\end{array}$ & Population Used in & $\begin{array}{l}\text { Dietary Assessment } \\
\text { Methods Used in } \\
\text { Publications }\end{array}$ & $\begin{array}{l}\text { Health-Related } \\
\text { Outcomes }\end{array}$ & Summary of Findings \\
\hline $\begin{array}{l}\text { Recommended Food } \\
\text { Score (RFS) }\end{array}$ & $\begin{array}{l}\text { Milte et al. } 2015 \text { [89] } \\
\text { Livingstone, } 2016 \text { [84] } \\
\text { Milte, } 2018 \text { [88] }\end{array}$ & Not tested & $\begin{array}{l}\text { Adults aged } \\
\text { 55-68 year }[88,89] \\
\geq 19 \text { years }[84]\end{array}$ & $\begin{array}{l}\text { 111-item FFQ and } \\
\text { food-related behavior } \\
\text { questions [88,89], } \\
\text { two 24-h Rs [84] }\end{array}$ & $\begin{array}{l}\text { Health-related } \\
\text { QOL [89]; obesity and } \\
\text { hypertension [84]; } \\
\text { Telomere length [88] }\end{array}$ & $\begin{array}{c}\text { Higher RFS scores were associated } \\
\text { with better health-related QOL } \\
\left(\mathrm{P}_{\text {trend }}<0.001\right)[89] \text { and less likely } \\
\text { to be hypertensive } \\
\left(\mathrm{P}_{\text {trend }}=0.021\right)[84] . \\
\text { No association between RFS and } \\
\text { telomere length [88]. }\end{array}$ \\
\hline $\begin{array}{l}\text { Not Recommended } \\
\text { Food Score (NRFS) }\end{array}$ & Petersen, 2015 [71] & Not tested & $\begin{array}{l}\text { Adults (mean } \\
\text { age }=50 \text { years) }[71]\end{array}$ & $\begin{array}{l}\text { FFQ (74-item food } \\
\text { and 6-item } \\
\text { alcohol) [71] }\end{array}$ & Diet quality [71] & $\begin{array}{l}\text { Mean NRFS scores for participants } \\
\text { with diabetes and controls were } \\
\text { not different [71]. }\end{array}$ \\
\hline $\begin{array}{l}\text { Mediterranean Diet } \\
\text { Score (MD Score) }\end{array}$ & $\begin{array}{l}\text { Petersen, } 2015[71] \\
\text { Dugue, } 2016[106] \\
\text { Hodge, } 2016[107] \\
\text { Hodge, } 2018[108]\end{array}$ & Not tested & $\begin{array}{c}\text { Adults (mean } \\
\text { age }=50 \text { years) [71], } \\
\text { aged } 27-76 \text { years }[106], \\
\text { mid-aged adults } \\
40-69 \text { years }[107,108]\end{array}$ & $\begin{array}{l}\text { FFQ (74-item food } \\
\text { and 6-item } \\
\text { alcohol) [71], 121-item } \\
\text { FFQ [106-108] }\end{array}$ & $\begin{array}{c}\text { Urothelial cell } \\
\text { carcinoma (UCC) } \\
\text { incidence [106]; lung } \\
\text { cancer [107]; total, } \\
\text { cardiovascular } \\
\text { disease (CVD), } \\
\text { coronary heart } \\
\text { disease (CHD) } \\
\text { mortality [108]; } \\
\text { Diet quality [71] }\end{array}$ & $\begin{array}{c}\text { Higher MD score was inversely } \\
\text { associated with invasive UCC (HR } \\
0.86 ; 95 \% \text { CI: } 0.74,1.00)[106] \\
\text { lung cancer risk }\left(\mathrm{HR}_{7-9} \text { vs. 0-3: } 0.64\right. \\
95 \% \text { CI: } 0.45,0.90)[107] \text { and total } \\
\text { mortality }\left(\mathrm{HR}_{\mathrm{Q} 5-\mathrm{Q} 1}: 0.86 ; 95 \% \mathrm{CI} \text { : }\right. \\
\text { 0.80, 0.93) [108]. } \\
\text { Mean MD scores for participants } \\
\text { with diabetes and controls were } \\
\text { not different [71]. }\end{array}$ \\
\hline $\begin{array}{l}\text { Mediterranean Diet } \\
\text { Pattern index (MDP } \\
\text { index) }\end{array}$ & Lai, 2016 [72] & Not tested & $\begin{array}{l}\text { Mid-aged women } \\
\text { (50-55 years) [72] }\end{array}$ & $\begin{array}{l}\text { FFQ (74-item food } \\
\text { and 6-item } \\
\text { alcohol) [72] }\end{array}$ & $\begin{array}{c}\text { Depressive } \\
\text { symptoms [72] }\end{array}$ & $\begin{array}{c}\text { Inverse association between MDP } \\
\text { index and depressive symptoms } \\
\left(P_{\text {trend }}=0.007\right)[72] .\end{array}$ \\
\hline MedDiet Score & Crichton, 2013 [115] & Not tested & $\begin{array}{l}\text { Adults aged } 40-65 \\
\text { years [115] }\end{array}$ & 215-item FFQ [115] & $\begin{array}{l}\text { Self-reported } \\
\text { psychological } \\
\text { functioning [115] }\end{array}$ & $\begin{array}{l}\text { Total MedDiet score was not } \\
\text { associated with cognitive function, } \\
\text { but plant food intakes was } \\
\text { beneficial for general health and } \\
\text { mental disorders }(p<0.05) \text { [115]. }\end{array}$ \\
\hline $\begin{array}{l}\text { Mediterranean Diet } \\
\text { Scale (MDS) }\end{array}$ & $\begin{array}{l}\text { Milte, } 2015 \text { [89] } \\
\text { Milte, } 2018 \text { [88] }\end{array}$ & Not tested & $\begin{array}{c}\text { Adults aged } 55-68 \\
\text { years }[88,89]\end{array}$ & $\begin{array}{l}111 \text {-item FFQ and } \\
\text { food-related behavior } \\
\text { questions }[88,89]\end{array}$ & $\begin{array}{l}\text { Health-related } \\
\text { QOL [89]; } \\
\text { Telomere length [88] }\end{array}$ & $\begin{array}{l}\text { Higher MDS scores were } \\
\text { associated with better } \\
\text { health-related QOL }(p<0.001) \text { [89] } \\
\text { No association between MDS and } \\
\text { relative telomere length [88]. }\end{array}$ \\
\hline
\end{tabular}


Table 2. Cont

\begin{tabular}{|c|c|c|c|c|c|c|}
\hline Index & Reference & $\begin{array}{l}\text { Validation Status of } \\
\text { Diet Quality Index }\end{array}$ & Population Used in & $\begin{array}{l}\text { Dietary Assessment } \\
\text { Methods Used in } \\
\text { Publications }\end{array}$ & $\begin{array}{l}\text { Health-Related } \\
\text { Outcomes }\end{array}$ & Summary of Findings \\
\hline $\begin{array}{l}\text { Dietary Approach to } \\
\text { Stop Hypertension } \\
\text { (DASH) }\end{array}$ & Petersen, 2015 [71] & Not tested & $\begin{array}{l}\text { Adults (mean } \\
\text { age }=50 \text { years) }[71]\end{array}$ & $\begin{array}{l}\text { FFQ (74-item food } \\
\text { and 6-item } \\
\text { alcohol) [71] }\end{array}$ & Diet quality [71] & $\begin{array}{l}\text { Mean DASH scores for } \\
\text { participants with diabetes and } \\
\text { controls were not different [71]. }\end{array}$ \\
\hline $\begin{array}{l}\text { Alternative Healthy } \\
\text { Eating Index (AHEI) }\end{array}$ & Petersen, 2015 [71] & Not tested & $\begin{array}{c}\text { Adults } \\
\text { (mean age }=50 \text { years) }[71]\end{array}$ & $\begin{array}{l}\text { FFQ (74-item food } \\
\text { and 6-item } \\
\text { alcohol) [71] }\end{array}$ & Diet quality [71] & $\begin{array}{l}\text { Mean AHEI scores for participants } \\
\text { with diabetes and controls were } \\
\text { not different [71]. }\end{array}$ \\
\hline $\begin{array}{l}\text { Alternative Healthy } \\
\text { Eating Index-2010 } \\
\text { (AHEI-2010) }\end{array}$ & Dugue, 2016 [106] & Not tested & $\begin{array}{l}\text { Adults aged 27-76 } \\
\text { years [106] }\end{array}$ & 121-item FFQ [106] & $\begin{array}{l}\text { Urothelial cell } \\
\text { carcinoma (UCC) } \\
\text { incidence [106] }\end{array}$ & $\begin{array}{c}\text { No association between } \\
\text { AHEI-2010 and risk of overall UCC } \\
\text { (HR: 1.03; 95\% CI: 0.92, 1.15) and } \\
\text { invasive UCC (HR: } 0.88 ; 95 \% \text { CI: } \\
0.75,1.04)[106] .\end{array}$ \\
\hline $\begin{array}{l}\text { Diet Quality } \\
\text { Tool (DQT) }\end{array}$ & O'Reilly, 2012 [32] & $\begin{array}{l}\text { Tested construct and } \\
\text { criterion validity [32] }\end{array}$ & $\begin{array}{l}\text { CVD patients (mean } \\
\text { age = } \\
61.2 \pm 10.8 \text { years) [32] }\end{array}$ & $\begin{array}{l}\text { 13-item questionnaire } \\
\text { from validated FFQ } \\
\text { and } 4 \text {-d food } \\
\text { diary [32] }\end{array}$ & Diet quality [32] & $\begin{array}{c}\text { Significant difference was found } \\
\text { between mean dietary fiber } \\
(p<0.05) \text { and } \% \text { total energy from } \\
\text { saturated fat }(p<0.01) \text { for those } \\
\text { with better DQT scores }(>60 \%) \text { vs. } \\
\text { poorer scores }(\leq 60 \%) \text { when } \\
\text { compared with } 4 \text {-day food diary } \\
\text { nutrient values [32]. }\end{array}$ \\
\hline
\end{tabular}


Table 2. Cont.

\begin{tabular}{|c|c|c|c|c|c|c|}
\hline Index & Reference & $\begin{array}{l}\text { Validation Status of } \\
\text { Diet Quality Index }\end{array}$ & Population Used in & $\begin{array}{l}\text { Dietary Assessment } \\
\text { Methods Used in } \\
\text { Publications }\end{array}$ & $\begin{array}{l}\text { Health-Related } \\
\text { Outcomes }\end{array}$ & Summary of Findings \\
\hline $\begin{array}{l}\text { Dietary Inflammatory } \\
\text { Index (DII) }\end{array}$ & $\begin{array}{c}\text { Wood, } 2015 \text { [114] } \\
\text { Dugue, } 2016 \text { [106] } \\
\text { Hodge, } 2016 \text { [107] } \\
\text { Shivappa, } 2016[111] \\
\text { Vissers, } 2016 \text { [113] } \\
\text { Vissers, } 2017 \text { [112] } \\
\text { Hodge, } 2018 \text { [108] } \\
\text { Mayr, } 2018 \text { [109] } \\
\text { Nagle, } 2019[110]\end{array}$ & $\begin{array}{c}\text { Tested construct } \\
\text { validity [66] }\end{array}$ & $\begin{array}{c}\text { Adults aged } \\
\geq 18 \text { years [110,114], } \\
\text { 27-76 years [106], } \\
\text { mid-aged adults } \\
\text { 40-69 years [107,108]; } \\
\text { mid-aged women } \\
\text { (50-55 years) [111-113]; } \\
\text { mean age-61.9 years [109] }\end{array}$ & $\begin{array}{c}\text { FFQ (74-item food } \\
\text { and 6-item } \\
\text { alcohol) [111-113], } \\
\text { 121-item } \\
\text { FFQ [106-108], } \\
\text { 139-item FFQ [110], } \\
\text { 186-item FFQ [114], } \\
\text { 7-day food diary [109] }\end{array}$ & $\begin{array}{c}\text { lung cancer [107]; } \\
\text { total, CVD, CHD } \\
\text { mortality [108]; } \\
\text { ovarian cancer risk } \\
\text { and survival [110]; } \\
\text { hypertension [112]; } \\
\text { CVD, CHD and } \\
\text { cerebrovascular } \\
\text { disease risk [113]; } \\
\text { Asthma risk [114]; } \\
\text { Interleukin 6 } \\
\text { (IL-6) [109]; } \\
\text { depression [111]; } \\
\text { UCC incidence [106] }\end{array}$ & 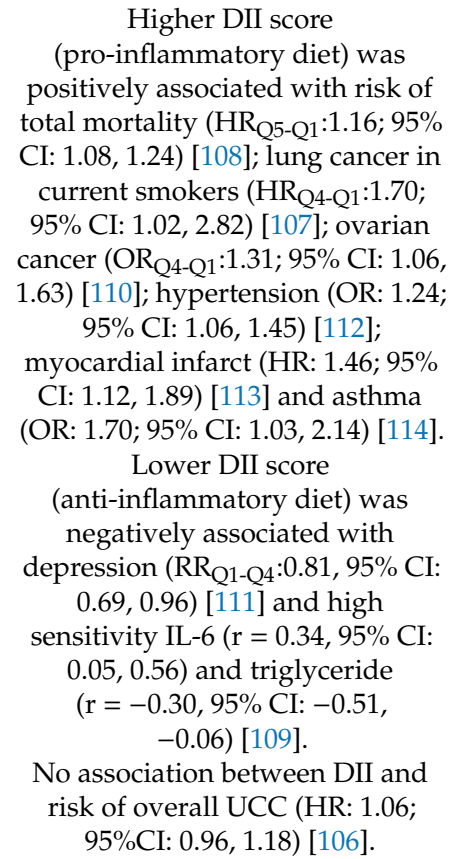 \\
\hline
\end{tabular}


Table 3. Critical appraisal of diet quality indices by previously suggested recommendations.

\begin{tabular}{|c|c|c|c|c|c|c|c|c|c|c|c|c|c|c|c|}
\hline & \multicolumn{2}{|c|}{$\begin{array}{l}\text { Theoretical } \\
\text { Framework }\end{array}$} & \multicolumn{4}{|c|}{ Dimension } & \multirow{2}{*}{$\begin{array}{c}\text { Structure } \\
\text { Nested/ } \\
\text { Ordered/ } \\
\text { Not } \\
\text { Ordered }\end{array}$} & \multicolumn{3}{|c|}{ Indicator Selection } & \multicolumn{3}{|c|}{ Scoring Criteria } & \multicolumn{2}{|c|}{ Aggregation } \\
\hline & $\begin{array}{l}\text { Dietary } \\
\text { Guideline }\end{array}$ & $\begin{array}{l}\text { Dietary } \\
\text { Pattern }\end{array}$ & Adequacy & Moderation & Variety & Balance & & Database & $\begin{array}{c}\text { Foods \& } \\
\text { Food } \\
\text { Groups/ } \\
\text { Nutrients/ } \\
\text { Both }\end{array}$ & $\begin{array}{l}\text { Healthy/ } \\
\text { Unhealthy } \\
\text { Component }\end{array}$ & $\begin{array}{l}\text { Dichotomous/ } \\
\text { Ordinal/ } \\
\text { Metric }\end{array}$ & Range & $\begin{array}{l}\text { Cut } \\
\text { Points }\end{array}$ & $\begin{array}{l}\text { Weighted } \\
\text { Equally } \\
\text { by } \\
\text { Indicators }\end{array}$ & $\begin{array}{l}\text { Evaluation of } \\
\text { DQI }\end{array}$ \\
\hline Aust-HEI [28] & Y & & Y & Y & Y & & $\begin{array}{c}\text { Not } \\
\text { ordered }\end{array}$ & $\begin{array}{c}\text { FFQ (item } \\
\text { not stated), } \\
\text { SDQ }\end{array}$ & $\begin{array}{c}\text { Foods \& } \\
\text { food } \\
\text { groups }\end{array}$ & Y & Ordinal & {$[0,60]$} & Y & Y & $\begin{array}{l}\text { Construct } \\
\text { Validity }\end{array}$ \\
\hline ARFS [29] & Y & & & & Y & & Ordered & $\begin{array}{l}\text { FFQ (74-item } \\
\text { food and } \\
\text { 6-item } \\
\text { alcohol) }\end{array}$ & $\begin{array}{c}\text { Foods \& } \\
\text { food } \\
\text { groups }\end{array}$ & $\mathrm{N}$ & Dichotomous & {$[0,74]$} & $\mathrm{N}$ & Y & $\begin{array}{l}\text { Construct } \\
\text { Validity }\end{array}$ \\
\hline ARFS-1 [42] & $\mathrm{Y}$ & & & & Y & & Ordered & $\begin{array}{c}\text { Subset of } 70 \\
\text { items from } \\
120 \text {-item FFQ }\end{array}$ & $\begin{array}{l}\text { Foods \& } \\
\text { food } \\
\text { groups }\end{array}$ & $\mathrm{N}$ & Dichotomous & {$[0,73]$} & $\mathrm{N}$ & Y & $\begin{array}{l}\text { Reproducibility, } \\
\text { comparative } \\
\text { validity }\end{array}$ \\
\hline DGI [19] & Y & & Y & Y & Y & & Nested & $\begin{array}{c}\text { 108-item } \\
\text { FFQ, } \\
\text { Single 24-h R }\end{array}$ & $\begin{array}{l}\text { Foods \& } \\
\text { food } \\
\text { groups }\end{array}$ & Y & Metric & {$[0,150]$} & Y & Y & $\begin{array}{l}\text { Construct } \\
\text { Validity }\end{array}$ \\
\hline $\begin{array}{l}\text { Modified } \\
\text { DGI [37] }\end{array}$ & Y & & Y & Y & Y & & Ordered & 137-item FFQ & $\begin{array}{l}\text { Foods \& } \\
\text { Food } \\
\text { groups }\end{array}$ & Y & Metric & {$[0,80]$} & Y & Y & Not tested \\
\hline DGI-2013 [17] & Y & & Y & Y & Y & & Nested & 111-item FFQ & $\begin{array}{c}\text { Foods \& } \\
\text { food } \\
\text { groups }\end{array}$ & $\mathrm{Y}$ & Metric & {$[0,130]$} & Y & Y & $\begin{array}{l}\text { Construct } \\
\text { Validity }\end{array}$ \\
\hline RDGI [36] & Y & & Y & $\mathrm{Y}$ & & & Nested & $\begin{array}{l}\text { 12-item FFQ, } \\
\text { 12-item DBQ }\end{array}$ & $\begin{array}{l}\text { Foods \& } \\
\text { food } \\
\text { groups }\end{array}$ & $\mathrm{Y}$ & Metric & {$[0,100]$} & Y & Y & Not tested \\
\hline $\begin{array}{c}\text { CSIRO } \\
\text { HDS [63] }\end{array}$ & Y & & $\mathrm{Y}$ & Y & Y & & Ordered & 38-item SFS & $\begin{array}{l}\text { Foods \& } \\
\text { food } \\
\text { groups }\end{array}$ & Y & Metric & {$[0,100]$} & Y & Y & $\begin{array}{l}\text { Relative } \\
\text { validity }\end{array}$ \\
\hline TDS [33] & Y & & Y & Y & Y & & Nested & 145-item FFQ & Both & $\mathrm{Y}$ & Ordinal & {$[0,20]$} & Y & Y & $\begin{array}{l}\text { Relative } \\
\text { validity }\end{array}$ \\
\hline Aussie-DQI [18] & ] $Y$ & & Y & Y & Y & & Ordered & $\begin{array}{l}\text { Single 24-h R, } \\
\text { 129-item FFQ }\end{array}$ & Both & Y & Metric & {$[0,120]$} & Y & Y & $\begin{array}{l}\text { Construct } \\
\text { Validity, } \\
\text { criterion } \\
\text { validity }\end{array}$ \\
\hline
\end{tabular}


Table 3. Cont.

\begin{tabular}{|c|c|c|c|c|c|c|c|c|c|c|c|c|c|c|c|}
\hline & \multicolumn{2}{|c|}{$\begin{array}{l}\text { Theoretical } \\
\text { Framework }\end{array}$} & \multicolumn{4}{|c|}{ Dimension } & \multirow{2}{*}{$\begin{array}{c}\text { Structure } \\
\text { Nested/ } \\
\text { Ordered/ } \\
\text { Not } \\
\text { Ordered }\end{array}$} & \multicolumn{3}{|c|}{ Indicator Selection } & \multicolumn{3}{|c|}{ Scoring Criteria } & \multicolumn{2}{|c|}{ Aggregation } \\
\hline & $\begin{array}{c}\text { Dietary } \\
\text { Guideline }\end{array}$ & $\begin{array}{l}\text { Dietary } \\
\text { Pattern }\end{array}$ & Adequacy & Moderation & Variety & Balance & & Database & $\begin{array}{c}\text { Foods \& } \\
\text { Food } \\
\text { Groups/ } \\
\text { Nutrients/ } \\
\text { Both }\end{array}$ & $\begin{array}{l}\text { Healthy/ } \\
\text { Unhealthy } \\
\text { Component }\end{array}$ & $\begin{array}{l}\text { Dichotomous/ } \\
\text { Ordinal/ } \\
\text { Metric }\end{array}$ & Range & $\begin{array}{c}\text { Cut } \\
\text { Points }\end{array}$ & $\begin{array}{c}\text { Weighted } \\
\text { Equally } \\
\text { by } \\
\text { Indicators }\end{array}$ & $\begin{array}{c}\text { Evaluation o } \\
\text { DQI }\end{array}$ \\
\hline HEIFA-2013 [16] & $\mathrm{Y}$ & & $\mathrm{Y}$ & $\mathrm{Y}$ & $\mathrm{Y}$ & & Nested & $\begin{array}{c}\text { Five 1-day } \\
\text { WFR, } \\
\text { FFQ (74-item } \\
\text { food and } \\
\text { 6-item } \\
\text { alcohol) } \\
\end{array}$ & Both & $\mathrm{Y}$ & Ordinal & {$[0,100]$} & $\mathrm{Y}$ & $\mathrm{Y}$ & $\begin{array}{l}\text { Internal } \\
\text { consistency } \\
\text { relative } \\
\text { validity }\end{array}$ \\
\hline ADQS [30] & $\mathrm{Y}$ & & $\mathrm{Y}$ & $\mathrm{Y}$ & & $\mathrm{Y}$ & $\begin{array}{c}\text { Not } \\
\text { ordered }\end{array}$ & $\begin{array}{l}\text { FFQ (74-item } \\
\text { food and } \\
\text { 6-item } \\
\text { alcohol) } \\
\end{array}$ & Both & $\mathrm{Y}$ & Metric & $\begin{array}{c}\text { Maximum } \\
= \\
\operatorname{RDI}(-10 \%)\end{array}$ & $\mathrm{Y}$ & $\mathrm{Y}$ & Not tested \\
\hline HDHI [20] & Y & & Y & Y & & & $\begin{array}{c}\text { Not } \\
\text { Ordered }\end{array}$ & $\begin{array}{l}\text { Multiple-pass } \\
\text { single 24-h R, } \\
\text { 25-item DHQ }\end{array}$ & $\begin{array}{l}\text { Foods \& } \\
\text { food } \\
\text { groups }\end{array}$ & $\mathrm{Y}$ & Ordinal & {$[0,60]$} & $\mathrm{Y}$ & $\mathrm{Y}$ & $\begin{array}{c}\text { Content } \\
\text { validity, } \\
\text { construct } \\
\text { validity, } \\
\text { Relative } \\
\text { validity }\end{array}$ \\
\hline DQI-R [8] & $\mathrm{Y}$ & & $\mathrm{Y}$ & $\mathrm{Y}$ & $\mathrm{Y}$ & & Ordered & Two 24-h Rs & Both & $\mathrm{Y}$ & Metric & {$[0,100]$} & Y & Y & $\begin{array}{c}\text { Concurrent } \\
\text { validity }\end{array}$ \\
\hline RFS [41] & $\mathrm{Y}$ & & $\mathrm{Y}$ & & $\mathrm{Y}$ & & Ordered & $\begin{array}{l}23 \text { items from } \\
62 \text {-item FFQ }\end{array}$ & $\begin{array}{l}\text { Foods \& } \\
\text { food } \\
\text { groups }\end{array}$ & $\mathrm{N}$ & Dichotomous & {$[0,23]$} & $\mathrm{N}$ & $\mathrm{Y}$ & Not tested \\
\hline NRFS [32] & $\mathrm{Y}$ & & & $\mathrm{Y}$ & & & $\begin{array}{c}\text { Not } \\
\text { ordered }\end{array}$ & $\begin{array}{c}\text { 60-item FFQ, } \\
\text { WFRs (days } \\
\text { not stated) }\end{array}$ & $\begin{array}{l}\text { Foods \& } \\
\text { food } \\
\text { groups }\end{array}$ & $\mathrm{N}$ & Dichotomous & {$[0,21]$} & $\mathrm{N}$ & $\mathrm{Y}$ & Not tested \\
\hline MD score [11] & & Y & Y & Y & & Y & $\begin{array}{c}\text { Not } \\
\text { ordered }\end{array}$ & 190-item FFQ & Both & Y & Dichotomous & {$[0,8]$} & $\mathrm{N}$ & Y & Not tested \\
\hline MDP index [21] & & $\mathrm{Y}$ & $\mathrm{Y}$ & $\mathrm{Y}$ & & $\mathrm{Y}$ & Ordered & 150-item FFQ & Both & $\mathrm{Y}$ & Dichotomous & {$[0,9]$} & $\mathrm{N}$ & $\mathrm{Y}$ & Not tested \\
\hline $\begin{array}{l}\text { MedDiet } \\
\text { score [47] }\end{array}$ & & $\mathrm{Y}$ & $\mathrm{Y}$ & $\mathrm{Y}$ & & & Ordered & 121-item FFQ & $\begin{array}{l}\text { Foods \& } \\
\text { food } \\
\text { groups }\end{array}$ & $\mathrm{Y}$ & Dichotomous & {$[0,9]$} & $\mathrm{N}$ & $\mathrm{Y}$ & Not tested \\
\hline
\end{tabular}


Table 3. Cont.

\begin{tabular}{|c|c|c|c|c|c|c|c|c|c|c|c|c|c|c|c|}
\hline & \multicolumn{2}{|c|}{$\begin{array}{l}\text { Theoretical } \\
\text { Framework }\end{array}$} & \multicolumn{4}{|c|}{ Dimension } & \multirow{2}{*}{$\begin{array}{c}\text { Structure } \\
\text { Nested/ } \\
\text { Ordered/ } \\
\text { Not } \\
\text { Ordered }\end{array}$} & \multicolumn{3}{|c|}{ Indicator Selection } & \multicolumn{3}{|c|}{ Scoring Criteria } & \multicolumn{2}{|c|}{ Aggregation } \\
\hline & $\begin{array}{c}\text { Dietary } \\
\text { Guideline }\end{array}$ & $\begin{array}{l}\text { Dietary } \\
\text { Pattern }\end{array}$ & Adequacy & Moderation & Variety & Balance & & Database & $\begin{array}{c}\text { Foods \& } \\
\text { Food } \\
\text { Groups/ } \\
\text { Nutrients/ } \\
\text { Both } \\
\end{array}$ & $\begin{array}{l}\text { Healthy/ } \\
\text { Unhealthy } \\
\text { Component }\end{array}$ & $\begin{array}{l}\text { Dichotomous/ } \\
\text { Ordinal/ } \\
\text { Metric }\end{array}$ & Range & $\begin{array}{c}\text { Cut } \\
\text { Points }\end{array}$ & $\begin{array}{l}\text { Weighted } \\
\text { Equally } \\
\text { by } \\
\text { Indicators }\end{array}$ & $\begin{array}{c}\text { Evaluation of } \\
\text { DQI }\end{array}$ \\
\hline MDS [39] & & $\mathrm{Y}$ & $\mathrm{Y}$ & $\mathrm{Y}$ & & $\mathrm{Y}$ & Ordered & $\begin{array}{c}\text { FFQ (item } \\
\text { not stated), } \\
\text { 24-h R (days } \\
\text { not stated) }\end{array}$ & Both & $\mathrm{Y}$ & Dichotomous & {$[0,9]$} & $\mathrm{N}$ & $\mathrm{Y}$ & Not tested \\
\hline DASH [15] & & $\mathrm{Y}$ & $\mathrm{Y}$ & $\mathrm{Y}$ & & & $\begin{array}{c}\text { Not } \\
\text { ordered }\end{array}$ & 116-item FFQ & Both & $\mathrm{Y}$ & Ordinal & {$[8,40]$} & $\mathrm{N}$ & $\mathrm{Y}$ & Not tested \\
\hline AHEI [23] & & $\mathrm{Y}$ & $\mathrm{Y}$ & $\mathrm{Y}$ & & $\mathrm{Y}$ & $\begin{array}{c}\text { Not } \\
\text { ordered }\end{array}$ & 130-item FFQ & Both & $\mathrm{Y}$ & Metric & {$[2.5,87.5]$} & $\mathrm{Y}$ & $Y^{*}$ & Not tested \\
\hline AHEI-2010 [22] & & $\mathrm{Y}$ & $\mathrm{Y}$ & $\mathrm{Y}$ & & Y & $\begin{array}{c}\text { Not } \\
\text { ordered }\end{array}$ & $\begin{array}{l}\text { FFQ (item } \\
\text { not stated) }\end{array}$ & Both & Y & Metric & {$[0,110]$} & $\mathrm{Y}$ & $\mathrm{Y}$ & Not tested \\
\hline DQT [32] & & $\mathrm{Y}$ & $\mathrm{Y}$ & $\mathrm{Y}$ & & & $\begin{array}{c}\text { Not } \\
\text { ordered }\end{array}$ & $\begin{array}{c}\text { 4-day FD, } \\
\text { 13-item } \\
\text { questionnaire }\end{array}$ & $\begin{array}{l}\text { Foods \& } \\
\text { food } \\
\text { groups }\end{array}$ & $\mathrm{Y}$ & Ordinal & {$[0,130]$} & $\mathrm{Y}$ & $\mathrm{Y}$ & $\begin{array}{l}\text { Construct and } \\
\text { criterion } \\
\text { validity }\end{array}$ \\
\hline DII [66] & & Y & $\mathrm{Y}$ & $\mathrm{Y}$ & Y & & $\begin{array}{c}\text { Not } \\
\text { ordered }\end{array}$ & $\begin{array}{l}\text { 7-day dietary } \\
\text { recalls, 24-h } \\
\text { Rs [67] }\end{array}$ & Both & Y & Metric & $\begin{array}{l}{[-8.87,} \\
+7.98]\end{array}$ & $\mathrm{N}$ & Y & $\begin{array}{c}\text { Construct } \\
\text { validity [67] }\end{array}$ \\
\hline
\end{tabular}

24-h R: 24-h recall; DBQ: dietary behavior questions; DHQ: dietary habit questionnaire; FD: food diary; FFQ: food frequency questionnaire; N: No; RDI: recommended daily intake; SDQ: short dietary questions; SFS: short food survey; WFR: weighted food record; Y: Yes. * valid for except multivitamin use. The preferable features of DQI in the table are highlighted. 


\section{Discussion}

This systematic review and critical appraisal summarizes 25 DQI represented in 76 papers, including Australian and New Zealand adults. When the Australian and New Zealand DQI were assessed by the recommended points suggested by the previous reviews, none of them met all suggested criteria. Almost half of the reviewed DQI were composed of recommended foods or food groups and nutrients, and based on metric indicators, as is recommended by Burggraf et al. [14]. Nearly one-quarter of DQI included adequacy, moderation, and balance, key dimensions outlined by Burggraf et al. (2018). However, only one-third of the DQI were constructed according to the preferred nested structure, i.e., constructed with hierarchy, allowing for in-depth analysis. As for the strengths of all the reviewed Australian and New Zealand DQI, components were equally weighted by indicators, and their construct and criterion validity had been evaluated. DGI, DGI-2013, TDS, HEIFA-2013 and Aussie-DQI performed best according to dimension and its structure, indicator selection, scoring criteria and evaluation.

Our review combines the recommendations from Burggraf et al. (2018) and Trijsburg et al. (2019), each of whom presented an inventory of DQI construction criteria using a range of international DQI. Only 11 of the 25 DQI used in Australian and New Zealand adults included in our review overlapped with those selected in the previous reviews, making ours the most comprehensive review of DQI for this region. Like DQI applicable in LMIC [13], separate scoring for healthy and unhealthy items was not reported in DQI used in Australian and New Zealand adults. In addition, similar to Burggraf et al. [14], most of the DQI used in Australian and New Zealand adults were constructed by focusing on health outcomes linked with overconsumption or over-nutrition. As suggested by the recent systematic review [13], it would be beneficial to develop a global DQ index covering both overand under-nutrition aspects for applying in different cross-countries settings.

Among the DQI based on Australian Dietary Guidelines, five indices were constructed with nested structure $[16,17,19,33,36]$; three indices is based on the latest Australian Dietary Guideline $[16,17,36]$, three indices being composed of metric scaling $[17,19,36]$ and evaluated among various age group ( $\geq 19$ years in DGI and Aussie-DQI, 55-65 years in DGI-2013, 63-83 years in TDS, 18-34 years in HEIFA-2013). For example, both DGI-2013 and HEIFA-2013 had similarities in terms of dimension, dimensional structure, cutoff points and evaluation, but differences in indicator selection (foods and food groups in DGI-2013, a combination of nutrients, foods and food groups in HEIFA-2013) and scaling (metric in DGI-2013, ordinal in HEIFA-2013). It would be advantageous to employ these indices for investigating the overall DQ of adults in longitudinal settings, similar to the work of Sotos-Prieto and colleagues [130]. The authors investigated the relationship between mortality and DQ assessed by three indices, which were different in description, composition and construction criteria [130].

Another DQ index appropriate for application in diet-health relationship studies is the Aussie-DQI constructed by Zarrin et al. [18]. It is an extensively evaluated DQ index by using two independent data sets for its development and validation. The evaluation of construct validity showed that higher Aussie-DQI scores were associated with gender, age, smoking status and body mass index [18]. Further, the criterion validity assessment demonstrated that there was a negative association between Aussie-DQI scores and cancer mortality among men [18]. Although most of its scoring was based on earlier Australian Dietary Guidelines [59,60], recommendations from the World Health Organization, United Kingdom and the United States of America were applied for intakes of processed meat, sugar and saturated fat. It would be beneficial to investigate the predictive ability of Aussie-DQI for morbidity and mortality in large population-based cohort studies.

Among DQI measuring specific dietary pattern recommendations, those based on a traditional Mediterranean diet had shown that beneficial effects on cancer, cardiovascular diseases and diabetes [131-133]. Nevertheless, these indices seem not to conform to the preferable features of DQI. They were generally developed with no nested structure, detailed scoring range with percentile cutoff points, and not evaluated for construct and criterion validity. It is intriguing to construct a Mediterranean diet-based index that meets with methodological finesse and applies to nutritional 
epidemiological studies [14]. In contrast, the AHEI-2010, which scores foods and nutrients that prevent chronic diseases based on the Mediterranean diet [48-52], seems to be more appropriate for application in diet-health relationship studies. The AHEI-2010 had promising features for dimensions (adequacy, moderation and balance), indicator selection (composed of a combination of nutrients, foods and food groups, with healthy and unhealthy components), metric scaling with normative cutoff points [22].

This review identified the available DQI used with Australian and New Zealand adults. All included studies were those that described the development, evaluation or validation and application of each DQ index for measuring the overall DQ of adults, especially in various Australian and New Zealand settings. The strength of the current review is that it applies newly defined criteria to critically appraise all existing DQI used in Australian and New Zealand adults. Two independent reviewers assessed eligibility and methodological quality and extracted data for the identified DQI. However, a limitation of this review is the use of only one database. Relevant DQI may have been missed if the papers did not report the words used in our search strategy. To avoid missing relevant DQI, our electronic database search was complemented by prescreening in a second database (EMBASE) and hand searching the reference lists of the retrieved papers, which yielded only one additional paper.

\section{Conclusions}

The development and application of DQI based on national nutritional guidelines, condition-specific recommendations or composition are expanding rapidly. Importantly, national dietary guidelines are updated periodically according to evidence-based information; and hence the development of DQI should be based on the recent dietary guidelines in order to capture the recent updates. Preferable features of DQ index such as theoretical framework, dimension, dimensional structure, indicator selection, scoring criteria, aggregation and its evaluation should be considered when applying DQI in diet-health relationship studies. While further work is needed to enhance the construction of all Australian and New Zealand DQI to bring them into alignment with recommended construction criteria, DGI, DGI-2013, TDS, HEIFA-2013 and Aussie-DQI performed relatively well.

Supplementary Materials: The following are available online at http://www.mdpi.com/2072-6643/12/12/3777/s1, Table S1 Quality assessment of studies (ADA Quality Criteria Checklist Primary Research); Table S2 Overview of components of diet quality indices; Table S3 Components and scoring of diet quality indices.

Author Contributions: The search strategy- developed by A.H., H.H.-H.; search- conducted by H.H.-H.; screening of the articles, methodological assessment and data extraction- done by H.H.-H. and K.P.; writing — original draft preparation, H.H.-H.; writing — review and editing, H.H.-H., A.H., K.P., M.T. and E.L.J. All authors have read and agreed to the published version of the manuscript.

Funding: This research received no external funding.

Acknowledgments: H.H.-H. was supported by the University of Newcastle Ph.D. scholarship. The author thanks Debbie Booth and Julie Mundy-Taylor for their support during the development of search strategies.

Conflicts of Interest: The authors declare no conflict of interest.

\section{References}

1. Waijers, P.M.C.M.; Feskens, E.J.M.; Ocke', M.C. A critical review of predefined diet quality scores. Br. J. Nutr. 2007, 97, 219-231. [CrossRef] [PubMed]

2. Arvaniti, F.; Panagiotakos, D.B. Healthy Indexes in Public Health Practice and Research: A Review. Crit. Rev. Food Sci. Nutr. 2008, 48, 317-327. [CrossRef] [PubMed]

3. Wirt, A.; Collins, C.E. Diet quality—What is it and does it matter? Public Health Nutr. 2009, 12, 2473-2492. [CrossRef] [PubMed]

4. Newby, P.K.; Tucker, K.L. Empirically derived eating patterns using factor or cluster analysis: A review. Nutr. Rev. 2004, 62, 177-203. [CrossRef] [PubMed]

5. Hu, F.B. Dietary pattern analysis: A new direction in nutritional epidemiology. Curr. Opin. Lipidol. 2002, 13, 3-9. [CrossRef] 
6. Kant, A.K. Dietary Patterns and Health Outcomes. J. Am. Diet. Assoc. 2004, 104, 615-635. [CrossRef]

7. Kourlaba, G.; Panagiotakos, D.B. Dietary quality indices and human health: A review. Maturitas 2009, 62, 1-8. [CrossRef]

8. Haines, P.S.; Siega-Riz, A.M.; Popkin, B.M. The Diet Quality Index Revised: A measurement instrument for populations. J. Am. Diet. Assoc. 1999, 99, 697-704. [CrossRef]

9. Kennedy, T.E.; Ohls, J.; Carlson, S.; Fleming, K. The healthy eating index: Design and applications. J. Am. Diet. Assoc. 1995, 95, 1103-1108. [CrossRef]

10. Patterson, R.E.; Haines, P.S.; Popkin, B.M. Diet quality index: Capturing a multidimensional behavior. J. Am. Diet. Assoc. 1994, 94, 57-64. [CrossRef]

11. Trichopoulou, A.; Kouris-Blazos, A.; Wahlqvist, M.L.; Gnardellis, C.; Lagiou, P.; Polychronopoulos, E.; Vassilakou, T.; Lipworth, L.; Trichopoulos, D. Diet and overall survival in elderly people. BMJ 1995, 311, 1457-1460. [CrossRef] [PubMed]

12. Fransen, H.P.; Ocke', M.C. Indices of diet quality. Curr. Opin. Clin. Nutr. Metab. Care 2008, 11, 559-565. [CrossRef] [PubMed]

13. Trijsburg, L.; Talsma, E.F.; De Vries, J.H.; Kennedy, G.; Kuijsten, A.; Brouwer, I.D. Diet quality indices for research in low-and middle-income countries: A systematic review. Nutr. Rev. 2019, 77, 515-540. [CrossRef] [PubMed]

14. Burggraf, C.; Teuber, R.; Brosig, S.; Meier, T. Review of a priori dietary quality indices in relation to their construction criteria. Nutr. Rev. 2018, 76, 747-764. [CrossRef] [PubMed]

15. Fung, T.T.; Chiuve, S.E.; McCullough, M.L.; Rexrode, K.M.; Logroscino, G.; Hu, F.B. Adherence to a DASH-style diet and risk of coronary heart disease and stroke in women. Arch. Intern. Med. 2008, 168, 713-720. [CrossRef] [PubMed]

16. Roy, R.; Hebden, L.; Rangan, A.; Allman-Farinelli, M. The development, application, and validation of a Healthy Eating Index for Australian Adults (HEIFA-2013). Nutrition 2016, 32, 432-440. [CrossRef]

17. Thorpe, M.G.; Milte, C.M.; Crawford, D.; McNaughton, S.A. A revised Australian Dietary Guideline Index and its association with key sociodemographic factors, health behaviors and body mass Index in peri-retirement aged adults. Nutrients 2016, 8, 160. [CrossRef]

18. Zarrin, R.; Ibiebele, T.I.; Marks, G.C. Development and validity assessment of a diet quality index for Australians. Asia Pac. J. Clin. Nutr. 2013, 22, 177-187. [CrossRef]

19. McNaughton, S.A.; Ball, K.; Crawford, D.; Mishra, G.D. An index of diet and eating patterns is a valid measure of diet quality in an Australian population. J. Nutr. 2008, 138, 86-93. [CrossRef]

20. Wong, J.E.; Haszard, J.J.; Howe, A.S.; Parnell, W.R.; Skidmore, P.M.L. Development of a Healthy Dietary Habits Index for New Zealand adults. Nutrients 2017, 9, 454. [CrossRef]

21. Trichopoulou, A.; Costacou, T.; Bamia, C.; Trichopoulos, D. Adherence to a Mediterranean Diet and Survival in a Greek Population. N. Engl. J. Med. 2003, 348, 2599-2608. [CrossRef] [PubMed]

22. Chiuve, S.E.; Fung, T.T.; Rimm, E.B.; Hu, F.B.; McCullough, M.L.; Wang, M.; Stampfer, M.J.; Willett, W.C. Alternative dietary indices both strongly predict risk of chronic disease. J. Nutr. 2012, 142, 1009-1018. [CrossRef] [PubMed]

23. McCullough, M.L.; Feskanich, D.; Stampfer, M.J.; Giovannucci, E.L.; Rimm, E.B.; Hu, F.B.; Spiegelman, D.; Hunter, D.J.; Colditz, G.A.; Willett, W.C. Diet quality and major chronic disease risk in men and women: Moving toward improved dietary guidance. Am. J. Clin. Nutr. 2002, 76, 1261-1271. [CrossRef]

24. Centre for Reviews and Dissemination. Systematic Reviews: CRD's Guidance for Undertaking Reviews in Health Care; CRD, University of York: York, UK, 2009.

25. Moher, D.; Liberati, A.; Tetzlaff, J.; Altman, D.G.; The PRISMA Group. Preferred Reporting Items for Systematic Reviews and Meta-Analyses: The PRISMA Statement. PLoS Med. 2009, 6, e1000097. [CrossRef] [PubMed]

26. Academy of Nutrition and Dietetics. Evidence Analysis Manual: Steps in the Academy Evidence Analysis Process; ADA Research and Strategic Business Development: Chicago, IL, USA, 2016.

27. Sambunjak, D.; Cumpston, M.; Watts, C. Module 4: Selecting Studies and Collecting Data. In Cochrane Interactive Learning: Conducting an Intervention Review; Cochrane. Available online: https://training.cochrane. org/interactivelearning/module-4-selecting-studies-and-collecting-data (accessed on 26 May 2019).

28. Australian Institute of Health and Welfare. Australian Diet Quality Index Project; Australian Institute of Health and Welfare: Canberra, Australia, 2007. 
29. Collins, C.E.; Young, A.F.; Hodge, A. Diet quality is associated with higher nutrient intake and self-rated health in mid-aged women. J. Am. Coll. Nutr. 2008, 27, 146-157. [CrossRef] [PubMed]

30. Froud, A.; Murphy, J.; Cribb, L.; Ng, C.H.; Sarris, J. The relationship between dietary quality, serum brain-derived neurotrophic factor (BDNF) level, and the Val66met polymorphism in predicting depression. Nutr. Neurosci. 2019, 22, 513-521. [CrossRef]

31. Michels, K.B.; Wolk, A. A prospective study of variety of healthy foods and mortality in women. Int. J. Epidemiol. 2002, 31, 847-854. [CrossRef]

32. O'Reilly, S.; McCann, L. Development and validation of the Diet Quality Tool for use in cardiovascular disease prevention settings. Aust. J. Prim. Health 2012, 18, 138-147. [CrossRef]

33. Russell, J.; Flood, V.; Rochtchina, E.; Gopinath, B.; Allman-Farinelli, M.; Bauman, A.; Mitchell, P. Adherence to dietary guidelines and 15-year risk of all-cause mortality. Br. J. Nutr. 2013, 109, 547-555. [CrossRef]

34. Shivappa, N.; Steck, S.E.; Hurley, T.G.; Hussey, J.R.; Hébert, J.R. Designing and developing a literature-derived, population-based dietary inflammatory index. Public Health Nutr. 2014, 17, 1689-1696. [CrossRef]

35. Williams, R.L.; Rollo, M.E.; Schumacher, T.; Collins, C.E. Diet quality scores of Australian adults who have completed the Healthy Eating Quiz. Nutrients 2017, 9, 880. [CrossRef] [PubMed]

36. Bivoltsis, A.; Trapp, G.; Knuiman, M.; Hooper, P.; Ambrosini, G. Can a simple dietary index derived from a sub-set of questionnaire items assess diet quality in a sample of Australian adults? Nutrients 2018, 10, 486. [CrossRef] [PubMed]

37. McLeod, E.R.; Campbell, K.J.; Hesketh, K.D. Nutrition knowledge: A mediator between socioeconomic position and diet quality in Australian first-time mothers. J. Am. Diet. Assoc. 2011, 111, 696-704. [CrossRef]

38. Hendrie, G.A.; Baird, D.; Golley, R.K.; Noakes, M. The CSIRO Healthy Diet Score: An online survey to estimate compliance with the Australian Dietary Guidelines. Nutrients 2017, 9, 47. [CrossRef] [PubMed]

39. Trichopoulou, A.; Orfanos, P.; Norat, T.; Bueno-de-Mesquita, B.; Ocké, M.; Peeters, P.H.; van der Schouw, Y.T.; Boeing, H.; Hoffmann, K.; Boffetta, P.; et al. Modified Mediterranean diet and survival: EPIC-elderly prospective cohort study. BMJ 2005, 330, 991-998. [CrossRef] [PubMed]

40. Kant, A.K.; Graubard, B.I. A comparison of three dietary pattern indexes for predicting biomarkers of diet and disease. J. Am. Coll. Nutr. 2005, 24, 294-303. [CrossRef]

41. Kant, A.K.; Schatzkin, A.; Graubard, B.I.; Schairer, C. A prospective study of diet quality and mortality in women. JAMA 2000, 283, 2109-2115. [CrossRef]

42. Collins, C.E.; Burrows, T.L.; Rollo, M.E.; Boggess, M.M.; Watson, J.F.; Guest, M.; Duncanson, K.; Pezdirc, K.; Hutchesson, M.J. The comparative validity and reproducibility of a diet quality index for adults: The Australian Recommended Food Score. Nutrients 2015, 7, 785-798. [CrossRef]

43. Ministry of Health (New Zealand). New Zealand Food and Nutrition Guidelines for Healthy Adults: A Background Paper; Ministry of Health (New Zealand): Wellington, New Zealand, 2003.

44. National Research Council: Committee on Diet and Health. Diet and Health: Implications for Reducing Chronic Disease Risk; National Academy Press: Washington, DC, USA, 1989. [CrossRef]

45. Yates, A.A.; Schlicker, S.A.; Suitor, C.W. Dietary reference intakes: The new basis for recommendations for calcium and related nutrients, B vitamins, and choline. J. Am. Diet. Assoc. 1998, 98, 699-706. [CrossRef]

46. Willett, W.C. Diet and health: What should we eat? Science 1994, 264, 532-537. [CrossRef]

47. Hodge, A.; English, D.; Itsiopoulos, C.; O'dea, K.; Giles, G. Does a Mediterranean diet reduce the mortality risk associated with diabetes: Evidence from the Melbourne Collaborative Cohort Study. Nutr. Metab. Cardiovasc. Dis. 2011, 21, 733-739. [CrossRef] [PubMed]

48. Willett, W.C.; Sacks, F.; Trichopoulou, A.; Drescher, G.; Ferro-Luzzi, A.; Helsing, E.; Trichopoulos, D. Mediterranean diet pyramid: A cultural model for healthy eating. Am. J. Clin. Nutr. 1995, 61, 1402S-1406S. [CrossRef] [PubMed]

49. Kushi, L.H.; Lenart, E.B.; Willett, W.C. Health implications of Mediterranean diets in light of contemporary knowledge. 2. Meat, wine, fats, and oils. Am. J. Clin. Nutr. 1995, 61, 1416S-1427S. [CrossRef]

50. Renaud, S.d.; de Lorgeril, M. Wine, alcohol, platelets, and the French paradox for coronary heart disease. Lancet 1992, 339, 1523-1526. [CrossRef]

51. de Lorderil, M.; Renaud, S.; Mamelle, N. Mediterranean alphalinolenic acid-rich diet in secondary prevention of cardiovascular disease. Lancet 1994, 343, 1454-1459. [CrossRef]

52. Kushi, L.H.; Lenart, E.B.; Willett, W.C. Health implications of Mediterranean diets in light of contemporary knowledge. 1. Plant foods and dairy products. Am. J. Clin. Nutr. 1995, 61, 1407S-1415S. [CrossRef] 
53. United States Department of Health and Human Services. Your Guide to Lowering Your Blood Pressure with DASH; National Institutes of Health, National Heart, Lung and Blood Institute: Bethesda, MD, USA, 2006.

54. National Heart Foundation of Australia and Cardiac Society of Australia and New Zealand. Reducing Risk in Heart Disease 2007: Guidelines for Preventing Cardiovascular Events in People with Coronary Heart Disease; National Heart Foundation of Australia: Canberra, Australia, 2008.

55. Kant, A.K.; Thompson, F.E. Measures of overall diet quality from a food frequency questionnaire: National Health Interview Survey, 1992. Nutr. Res. 1997, 17, 1443-1456. [CrossRef]

56. Wahlqvist, M.L.; Lo, C.S.; Myers, K.A. Food variety is associated with less macrovascular disease in those with type II diabetes and their healthy controls. J. Am. Coll. Nutr. 1989, 8, 515-523. [CrossRef]

57. Newby, P.; Hu, F.B.; Rimm, E.B.; Smith-Warner, S.A.; Feskanich, D.; Sampson, L.; Willett, W.C. Reproducibility and validity of the Diet Quality Index Revised as assessed by use of a food-frequency questionnaire. Am. J. Clin. Nutr. 2003, 78, 941-949. [CrossRef]

58. Fogli-Cawley, J.J.; Dwyer, J.T.; Saltzman, E.; McCullough, M.L.; Troy, L.M.; Jacques, P.F. The 2005 dietary guidelines for Americans adherence index: Development and application. J. Nutr. 2006, 136, 2908-2915. [CrossRef]

59. Kellett, E.; Smith, A.; Schmerlaib, Y. Australian Guide to Healthy Eating; Commonwealth Department of Health and Family Services: Canberra, Australia, 1998.

60. National Health and Medical Research Council (Australia). Dietary Guidelines for Australian Adults, 3rd ed.; National Health and Medical Research Council: Canberra, Australia, 2003.

61. National Health and Medical Research Council (Australia). Australian Dietary Guidelines; National Health and Medical Research Council: Canberra, Australia, 2013.

62. Marshall, S.; Watson, J.; Burrows, T.; Guest, M.; Collins, C.E. The development and evaluation of the Australian child and adolescent recommended food score: A cross-sectional study. Nutr. J. 2012, 11. [CrossRef]

63. Hendrie, G.A.; Rebuli, M.A.; Golley, R.K. Reliability and relative validity of a diet index score for adults derived from a self-reported short food survey. Nutr. Diet. 2017, 74, 291-297. [CrossRef]

64. Australian Bureau of Statistics. National Health Priority Areas. Available online: https://www.abs. gov.au/ausstats/abs@.nsf/Products/5317BAD6B8EEE19ACA25757C001EED30?opendocument (accessed on 21 March 2020).

65. Wong, J.E.; Skidmore, P.M.; Williams, S.M.; Parnell, W.R. Healthy dietary habits score as an indicator of diet quality in New Zealand adolescents. J. Nutr. 2014, 144, 937-942. [CrossRef]

66. Shivappa, N.; Steck, S.E.; Hurley, T.G.; Hussey, J.R.; Ma, Y.; Ockene, I.S.; Tabung, F.; Hébert, J.R. A population-based dietary inflammatory index predicts levels of C-reactive protein in the Seasonal Variation of Blood Cholesterol Study (SEASONS). Public Health Nutr. 2014, 17, 1825-1833. [CrossRef]

67. Cavicchia, P.P.; Steck, S.E.; Hurley, T.G.; Hussey, J.R.; Ma, Y.; Ockene, I.S.; Hébert, J.R. A new dietary inflammatory index predicts interval changes in serum high-sensitivity C-reactive protein. J. Nutr. 2009, 139, 2365-2372. [CrossRef]

68. Russell, J.C.; Flood, V.M.; Sadeghpour, A.; Gopinath, B.; Mitchell, P. Total Diet Score as a valid method of measuring diet quality among older adults. Asia Pac. J. Clin. Nutr. 2017, 26, 212-219. [CrossRef]

69. Thompson, F.E.; Subar, A.F. Dietary assessment methodology. In Nutrition in the Prevention and Treatment of Disease; Elsevier: Amsterdam, The Netherlands, 2017; pp. 5-48.

70. Alhazmi, A.; Stojanovski, E.; McEvoy, M.; Brown, W.; Garg, M.L. Diet quality score is a predictor of type 2 diabetes risk in women: The Australian Longitudinal Study on Women's Health. Br. J. Nutr. 2014, 112, 945-951. [CrossRef]

71. Petersen, K.S.; Blanch, N.; Wepener, R.H.; Clifton, P.M.; Keogh, J.B. Dietary quality in people with type 1 and type 2 diabetes compared to age, sex and BMI matched controls. Diabetes Res. Clin. Pract. 2015, 107, e7-e10. [CrossRef]

72. Lai, J.S.; Oldmeadow, C.; Hure, A.J.; McEvoy, M.; Byles, J.; Attia, J. Longitudinal diet quality is not associated with depressive symptoms in a cohort of middle-aged Australian women. Br. J. Nutr. 2016, 115, 842-850. [CrossRef]

73. Lai, J.S.; Hure, A.J.; Oldmeadow, C.; McEvoy, M.; Byles, J.; Attia, J. Prospective study on the association between diet quality and depression in mid-aged women over 9 years. Eur. J. Nutr. 2017, 56, 273-281. [CrossRef] 
74. Aljadani, H.M.A.; Sibbritt, D.; Patterson, A.; Clare, C. The Australian Recommended Food Score did not predict weight gain in middle-aged Australian women during six years of follow-up. Aust. N. Z. J. Public Health 2013, 37, 322-328. [CrossRef]

75. Aljadani, H.A.; Patterson, A.; Sibbritt, D.; Hutchesson, M.J.; Jensen, M.E.; Collins, C.E. Diet quality measured by fruit and vegetable intake predicts weight change in young women. J. Obes. 2013, 2013, 525161. [CrossRef]

76. Aljadani, H.M.; Patterson, A.J.; Sibbritt, D.; Collins, C.E. Diet quality and 6-year risk of overweight and obesity among mid-age Australian women who were initially in the healthy weight range. Health Promot. J. Austr. 2016, 27, 29-35. [CrossRef]

77. Morrison, M.; Koh, D.; Lowe, J.; Miller, Y.D.; Marshall, A.L.; Colyvas, K.; Collins, C. Postpartum diet quality in Australian women following a gestational diabetes pregnancy. Eur. J. Clin. Nutr. 2012, 66, 1160-1165. [CrossRef]

78. Potter, J.; Collins, C.; Brown, L.; Hure, A. Diet quality of Australian breast cancer survivors: A cross-sectional analysis from the Australian Longitudinal Study on Women's Health. J. Hum. Nutr. Diet. 2014, 27, 569-576. [CrossRef] [PubMed]

79. Collins, C.E.; Patterson, A.; Fitzgerald, D. Higher diet quality does not predict lower Medicare costs but does predict number of claims in mid-aged Australian women. Nutrients 2011, 3, 40-48. [CrossRef]

80. Kullen, C.J.; Farrugia, J.-L.; Prvan, T.; O'Connor, H.T. Relationship between general nutrition knowledge and diet quality in Australian military personnel. Br. J. Nutr. 2016, 115, 1489-1497. [CrossRef]

81. Leech, R.M.; Livingstone, K.M.; Worsley, A.; Timperio, A.; McNaughton, S.A. Meal frequency but not snack frequency is associated with micronutrient intakes and overall diet quality in Australian men and women. J. Nutr. 2016, 146, 2027-2034. [CrossRef]

82. Leech, R.M.; Timperio, A.; Livingstone, K.M.; Worsley, A.; McNaughton, S.A. Temporal eating patterns: Associations with nutrient intakes, diet quality, and measures of adiposity. Am. J. Clin. Nutr. 2017, 106, 1121-1130. [CrossRef]

83. Livingstone, K.; Olstad, D.; Leech, R.; Ball, K.; Meertens, B.; Potter, J.; Cleanthous, X.; Reynolds, R.; McNaughton, S. Socioeconomic inequities in diet quality and nutrient intakes among Australian adults: Findings from a nationally representative cross-sectional study. Nutrients 2017, 9, 1092. [CrossRef]

84. Livingstone, K.M.; McNaughton, S.A. Diet quality is associated with obesity and hypertension in Australian adults: A cross sectional study. BMC Public Health 2016, 16, 1037. [CrossRef]

85. Livingstone, K.M.; McNaughton, S.A. Association between diet quality, dietary patterns and cardiometabolic health in Australian adults: A cross-sectional study. Nutr. J. 2018, 17, 19. [CrossRef]

86. Martin, J.; Moran, L.; Teede, H.; Ranasinha, S.; Lombard, C.; Harrison, C. Exploring diet quality between urban and rural dwelling women of reproductive age. Nutrients 2017, 9, 586. [CrossRef]

87. Martin, J.; Moran, L.; Teede, H.; Ranasinha, S.; Lombard, C.; Harrison, C. Diet quality in a weight gain prevention trial of reproductive aged women: A secondary analysis of a cluster randomized controlled trial. Nutrients 2019, 11, 49. [CrossRef]

88. Milte, C.M.; Russell, A.P.; Ball, K.; Crawford, D.; Salmon, J.; McNaughton, S.A. Diet quality and telomere length in older Australian men and women. Eur. J. Nutr. 2018, 57, 363-372. [CrossRef]

89. Milte, C.M.; Thorpe, M.G.; Crawford, D.; Ball, K.; McNaughton, S.A. Associations of diet quality with health-related quality of life in older Australian men and women. Exp. Gerontol. 2015, 64, 8-16. [CrossRef]

90. Ribeiro, R.V.; Hirani, V.; Senior, A.M.; Gosby, A.K.; Cumming, R.G.; Blyth, F.M.; Naganathan, V.; Waite, L.M.; Handelsman, D.J.; Kendig, H. Diet quality and its implications on the cardio-metabolic, physical and general health of older men: The Concord Health and Ageing in Men Project (CHAMP). Br. J. Nutr. 2017, 118, 130-143. [CrossRef] [PubMed]

91. Thorpe, M.G.; Kestin, M.; Riddell, L.J.; Keast, R.S.; McNaughton, S.A. Diet quality in young adults and its association with food-related behaviours. Public Health Nutr. 2013, 17, 1767-1775. [CrossRef]

92. Gopinath, B.; Russell, J.; Flood, V.M.; Burlutsky, G.; Mitchell, P. Adherence to dietary guidelines positively affects quality of life and functional status of older adults. J. Acad. Nutr. Diet. 2014, 114, 220-229. [CrossRef]

93. Gopinath, B.; Russell, J.; Kifley, A.; Flood, V.M.; Mitchell, P. Adherence to dietary guidelines and successful aging over 10 years. J. Gerontol. A Biol. Sci. Med. Sci. 2016, 71, 349-355. [CrossRef]

94. Hong, T.; Flood, V.; Rochtchina, E.; Mitchell, P.; Russell, J.; Wang, J.J. Adherence to dietary guidelines and the 10-year cumulative incidence of visual impairment: The Blue Mountains Eye Study. Am. J. Ophthalmol. 2014, 158, 302-308. [CrossRef] [PubMed] 
95. Gopinath, B.; Flood, V.M.; Wang, J.J.; Rochtchina, E.; Wong, T.Y.; Mitchell, P. Is quality of diet associated with the microvasculature? An analysis of diet quality and retinal vascular calibre in older adults. Br. J. Nutr. 2013, 110, 739-746. [CrossRef] [PubMed]

96. Gopinath, B.; Harris, D.; Flood, V.; Burlutsky, G.; Mitchell, P. A better diet quality is associated with a reduced likelihood of CKD in older adults. Nutr. Metab. Cardiovasc. Dis. 2013, 23, 937-943. [CrossRef] [PubMed]

97. Gopinath, B.; Rochtchina, E.; Flood, V.; Mitchell, P. Diet quality is prospectively associated with incident impaired fasting glucose in older adults. Diabet. Med. 2013, 30, 557-562. [CrossRef]

98. Gopinath, B.; Schneider, J.; Flood, V.; McMahon, C.; Burlutsky, G.; Leeder, S.; Mitchell, P. Association between diet quality with concurrent vision and hearing impairment in older adults. J. Nutr. Health Aging 2014, 18, 251-256. [CrossRef] [PubMed]

99. Roach, L.A.; Lambert, K.; Holt, J.L.; Meyer, B.J. Diet quality in patients with end-stage kidney disease undergoing dialysis. J Ren Care 2017, 43, 226-234. [CrossRef] [PubMed]

100. McNaughton, S.A.; Dunstan, D.W.; Ball, K.; Shaw, J.; Crawford, D. Dietary quality Is associated with diabetes and cardio-metabolic risk factors. J. Nutr. 2009, 139, 734-742. [CrossRef]

101. Arabshahi, S.; Lahmann, P.H.; Williams, G.M.; Marks, G.C.; van der Pols, J.C. Longitudinal change in diet quality in Australian adults varies by demographic, socio-economic, and lifestyle characteristics. J. Nutr. 2011, 141, 1871-1879. [CrossRef]

102. Arabshahi, S.; van der Pols, J.C.; Williams, G.M.; Marks, G.C.; Lahmann, P.H. Diet quality and change in anthropometric measures: 15-year longitudinal study in Australian adults. Br. J. Nutr. 2012, 107, 1376-1385. [CrossRef]

103. Backholer, K.; Spencer, E.; Gearon, E.; Magliano, D.J.; McNaughton, S.A.; Shaw, J.E.; Peeters, A. The association between socio-economic position and diet quality in Australian adults. Public Health Nutr. 2016, 19, 477-485. [CrossRef]

104. Olstad, D.L.; Lamb, K.E.; Thornton, L.E.; McNaughton, S.A.; Crawford, D.A.; Minaker, L.M.; Ball, K. Prospective associations between diet quality and body mass index in disadvantaged women: The Resilience for Eating and Activity Despite Inequality (READI) study. Int. J. Epidemiol. 2017, 46, 1433-1443. [CrossRef] [PubMed]

105. Smith, K.J.; McNaughton, S.A.; Gall, S.L.; Otahal, P.; Dwyer, T.; Venn, A.J. Associations between partnering and parenting transitions and dietary habits in young adults. J. Acad. Nutr. Diet. 2017, 117, 1210-1221. [CrossRef]

106. Dugué, P.A.; Hodge, A.M.; Brinkman, M.T.; Bassett, J.K.; Shivappa, N.; Hebert, J.R.; Hopper, J.L.; English, D.R.; Milne, R.L.; Giles, G.G. Association between selected dietary scores and the risk of urothelial cell carcinoma: A prospective cohort study. Int. J. Cancer 2016, 139, 1251-1260. [CrossRef] [PubMed]

107. Hodge, A.; Bassett, J.; Shivappa, N.; Hébert, J.; English, D.; Giles, G.; Severi, G. Dietary inflammatory index, Mediterranean diet score, and lung cancer: A prospective study. Cancer Causes Control 2016, 27, 907-917. [CrossRef] [PubMed]

108. Hodge, A.M.; Bassett, J.K.; Dugué, P.-A.; Shivappa, N.; Hébert, J.R.; Milne, R.; English, D.R.; Giles, G.G. Dietary inflammatory index or Mediterranean diet score as risk factors for total and cardiovascular mortality. Nutr. Metab. Cardiovasc. Dis. 2018, 28, 461-469. [CrossRef] [PubMed]

109. Mayr, H.L.; Itsiopoulos, C.; Tierney, A.C.; Ruiz-Canela, M.; Hebert, J.R.; Shivappa, N.; Thomas, C.J. Improvement in dietary inflammatory index score after 6-month dietary intervention is associated with reduction in interleukin-6 in patients with coronary heart disease: The AUSMED heart trial. Nutr. Res. 2018, 55, 108-121. [CrossRef] [PubMed]

110. Nagle, C.; Ibiebele, T.; Shivappa, N.; Hébert, J.; DeFazio, A.; Webb, P.; Study, A.O.C. The association between the inflammatory potential of diet and risk of developing, and survival following, a diagnosis of ovarian cancer. Eur. J. Nutr. 2019, 58, 1747-1756. [CrossRef]

111. Shivappa, N.; Schoenaker, D.A.J.M.; Hebert, J.R.; Mishra, G.D. Association between inflammatory potential of diet and risk of depression in middle-aged women: The Australian Longitudinal Study on Women's Health. Br. J. Nutr. 2016, 116, 1077-1086. [CrossRef]

112. Vissers, L.E.T.; Waller, M.; van der Schouw, Y.T.; Hébert, J.R.; Shivappa, N.; Schoenaker, D.A.J.M.; Mishra, G.D. A pro-inflammatory diet is associated with increased risk of developing hypertension among middle-aged women. Nutr. Metab. Cardiovasc. Dis. 2017, 27, 564-570. [CrossRef] 
113. Vissers, L.E.T.; Waller, M.A.; van der Schouw, Y.T.; Hebert, J.R.; Shivappa, N.; Schoenaker, D.A.J.M.; Mishra, G.D. The relationship between the dietary inflammatory index and risk of total cardiovascular disease, ischemic heart disease and cerebrovascular disease: Findings from an Australian population-based prospective cohort study of women. Atherosclerosis 2016, 253, 164-170. [CrossRef]

114. Wood, L.G.; Shivappa, N.; Berthon, B.S.; Gibson, P.G.; Hebert, J.R. Dietary inflammatory index is related to asthma risk, lung function and systemic inflammation in asthma. Clin. Exp. Allergy 2015, 45, 177-183. [CrossRef] [PubMed]

115. Crichton, G.E.; Bryan, J.; Hodgson, J.M.; Murphy, K.J. Mediterranean diet adherence and self-reported psychological functioning in an Australian sample. Appetite 2013, 70, 53-59. [CrossRef] [PubMed]

116. Reeves, M.M.; Healy, G.N.; Owen, N.; Shaw, J.E.; Zimmet, P.Z.; Dunstan, D.W. Joint associations of poor diet quality and prolonged television viewing time with abnormal glucose metabolism in Australian men and women. Prev. Med. 2013, 57, 471-476. [CrossRef] [PubMed]

117. Grech, A.; Sui, Z.; Siu, H.Y.; Zheng, M.; Allman-Farinelli, M.; Rangan, A. Socio-demographic determinants of diet quality in Australian adults using the validated Healthy Eating Index for Australian Adults (HEIFA-2013). Health Care (Don Mills) 2017, 5, 7. [CrossRef] [PubMed]

118. Harbury, C.; Collins, C.E.; Callister, R. Diet quality is lower among adults with a BMI $\geq 40 \mathrm{~kg} \mathrm{~m}^{-2}$ or a history of weight loss surgery. Obes. Res. Clin. Pract. 2019, 13, 197-204. [CrossRef] [PubMed]

119. Roy, R.; Rangan, A.; Hebden, L.; Louie, J.C.Y.; Tang, L.M.; Kay, J.; Allman-Farinelli, M. Dietary contribution of foods and beverages sold within a university campus and its effect on diet quality of young adults. Nutrition 2017, 34, 118-123. [CrossRef]

120. Forsyth, A.K.; Williams, P.G.; Deane, F.P. Nutrition status of primary care patients with depression and anxiety. Aust. J. Prim. Health 2012, 18, 172-176. [CrossRef]

121. Forsyth, A.; Deane, F.P.; Williams, P. A lifestyle intervention for primary care patients with depression and anxiety: A randomised controlled trial. Psychiatry Res. 2015, 230, 537-544. [CrossRef]

122. Baker, A.L.; Turner, A.; Kelly, P.J.; Spring, B.; Callister, R.; Collins, C.E.; Woodcock, K.L.; Kay-Lambkin, F.J.; Devir, H.; Lewin, T.J. 'Better Health Choices' by telephone: A feasibility trial of improving diet and physical activity in people diagnosed with psychotic disorders. Psychiatry Res. 2014, 220, 63-70. [CrossRef]

123. O’Brien, K.M.; Hutchesson, M.J.; Jensen, M.; Morgan, P.; Callister, R.; Collins, C.E. Participants in an online weight loss program can improve diet quality during weight loss: A randomized controlled trial. Nutr. J. 2014, 13, 82. [CrossRef]

124. Ashton, L.; Williams, R.; Wood, L.; Schumacher, T.; Burrows, T.; Rollo, M.; Pezdirc, K.; Callister, R.; Collins, C. Comparison of Australian Recommended Food Score (ARFS) and plasma carotenoid concentrations: A validation study in adults. Nutrients 2017, 9, 888. [CrossRef] [PubMed]

125. Ashton, L.M.; Morgan, P.J.; Hutchesson, M.J.; Rollo, M.E.; Collins, C.E. Feasibility and preliminary efficacy of the 'HEYMAN'healthy lifestyle program for young men: A pilot randomised controlled trial. Nutr. J. 2017, 16, 2. [CrossRef] [PubMed]

126. Ashton, L.; Pezdirc, K.; Hutchesson, M.; Rollo, M.; Collins, C. Is skin coloration measured by reflectance spectroscopy related to intake of nutrient-dense foods? A cross-sectional evaluation in Australian young adults. Nutrients 2018, 10, 11. [CrossRef] [PubMed]

127. Hendrie, G.; Golley, R.; Noakes, M. Compliance with dietary guidelines varies by weight status: A cross-sectional study of Australian adults. Nutrients 2018, 10, 197. [CrossRef] [PubMed]

128. Grech, A.; Rangan, A.; Allman-Farinelli, M. Social determinants and poor diet quality of energy-dense diets of Australian young adults. Health Care (Don Mills) 2017, 5, 70. [CrossRef]

129. Davison, B.; Saeedi, P.; Black, K.; Harrex, H.; Haszard, J.; Meredith-Jones, K.; Quigg, R.; Skeaff, S.; Stoner, L.; Wong, J. The association between parent diet quality and child dietary patterns in nine-to eleven-year-old children from Dunedin, New Zealand. Nutrients 2017, 9, 483. [CrossRef]

130. Sotos-Prieto, M.; Bhupathiraju, S.N.; Mattei, J.; Fung, T.T.; Li, Y.; Pan, A.; Willett, W.C.; Rimm, E.B.; Hu, F.B. Association of Changes in Diet Quality with Total and Cause-Specific Mortality. N. Engl. J. Med. 2017, 377, 143-153. [CrossRef]

131. Schwingshackl, L.; Hoffmann, G. Diet Quality as Assessed by the Healthy Eating Index, the Alternate Healthy Eating Index, the Dietary Approaches to Stop Hypertension Score, and Health Outcomes: A Systematic Review and Meta-Analysis of Cohort Studies. J. Acad. Nutr. Diet. 2015, 115, 780-800. [CrossRef] 
132. Schwingshackl, L.; Bogensberger, B.; Hoffmann, G. Diet Quality as Assessed by the Healthy Eating Index, Alternate Healthy Eating Index, Dietary Approaches to Stop Hypertension Score, and Health Outcomes: An Updated Systematic Review and Meta-Analysis of Cohort Studies. J. Acad. Nutr. Diet. 2018, 118, 74-100. [CrossRef]

133. Shen, J.; Wilmot, K.A.; Ghasemzadeh, N.; Molloy, D.L.; Burkman, G.; Mekonnen, G.; Gongora, M.C.; Quyyumi, A.A.; Sperling, L.S. Mediterranean Dietary Patterns and Cardiovascular Health. Annu. Rev. Nutr. 2015, 35, 425-449. [CrossRef]

Publisher's Note: MDPI stays neutral with regard to jurisdictional claims in published maps and institutional affiliations.

(C) 2020 by the authors. Licensee MDPI, Basel, Switzerland. This article is an open access article distributed under the terms and conditions of the Creative Commons Attribution (CC BY) license (http://creativecommons.org/licenses/by/4.0/). 